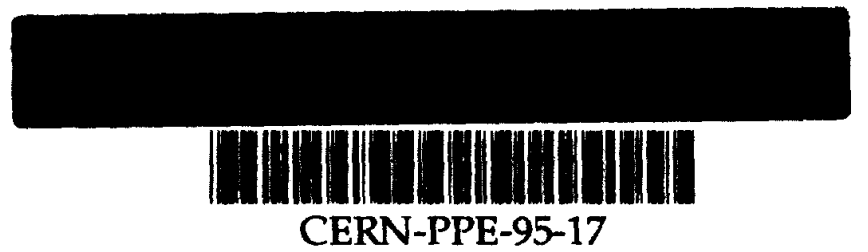

L. (ERN-PPE/95-(017

16 February 1995

CERN-PPE-95-17

sw 9511

\title{
SICAL - a high precision silicon-tungsten luminosity calorimeter for ALEPH
}

D. Bédérède. E. Beuville, B. Bloch-Devaux, L. Gosset, A. Joudon, E. Lançon, J. Pascual, J. Perlas ${ }^{1}$, B. Peyaud, J. Rander, J.F. Renardy, J.P. Schuller, B. Vallage

Service de Physique des Particules, DAPNIA. CE-Saclay, 91191 Gif-sur-Yvette Ceder. France ${ }^{2}$

R. Benetta, J. Boudreau ${ }^{3}$, J. Griffiths, R. Grabit. P. Jarron. B. Lofstedt.

R. Miquel, J.C. Santiard, G. Stefanini, H. Wahl

Europen Laboratory for Particle Physics (CERN), 1211 Cieneva 23. Surtzerland

C. Avanzini, C. Cerri, R. Fantechi, R. Lorenzini ${ }^{4}$, E.B. Martin ${ }^{5}$, G. Pierazzini, A. Valassi

Dipartimento di Fisica dell'Università, INFN Sezione di Pisa, e Scuola Normale Superiore. 56010 Pisa, Italy

\begin{abstract}
The design, construction and performance of a silicon-tungsten electromagnetic calorimeter built to achieve an experimental luminosity measurement precision at LEP $\leq 0.1 \%$ is described. The detector uses homogeneous construction to give full azimuthal acceptance for Bhabha scattering over polar angles from 24 to $58 \mathrm{mrad}$. Detailed information concerning shower developement is obtained from zero-suppressed readout of the 12288 pads of the detector. Trigger decisions are gencrated from a rapid flash-ADC system using programmable gate arrays.
\end{abstract}

Submitted to Nucl. Instrum. Methods

\footnotetext{
1Now at Institut de Fisica d'Altes Energies, Iniversitat Autonoma de Barcelona. Spain

"Supported by the Direction des Sciences de la Matière. C.E.t. France

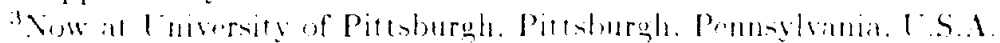

Now at ENEA. Palermo, Italy

¿Now at ('ERX. lell (ieneva 23. Switzerland
} 


\section{Introduction}

Bhabha scattering $\left(e^{+} e^{-} \rightarrow e^{+} e^{-}\right)$has been generally used as the reference reaction in $\epsilon^{+} \epsilon^{-}$ experiments. The rate of such events detected in specially designed monitors is used to measure the absolute luminosity of the colliding beams, found by dividing the number of monitor events by the Bhabha cross section integrated over the acceptance. The cross sections for processes studied in a colliding-beam detector are then determined with respect to the absolute luminosity given by the reference reaction. At LEP energies this technique is used in precision measurements of the $Z$ resonance cross sections. which are essential for the determination of the number of light neutrino species. as well as the $Z$ parameters. The electroweak process $Z \rightarrow+^{+}{ }^{-}$creates an interference for such a reference. however. the effect can be limited by restricting the acceptance to low angles where the Bhabha rates are dominated by small values of four-momentum transfer and therefore largely described by quantum electrodynamics (QED). The large cross-section at low angles gives a further advantage of reducing the statistical uncertainty of the reference measurement.

After the first round of electroweak measurements at LEP, a goal of $0.1 \%$ was fixed for the experimental precision of the ALEPH experiment's [1] luminosity measurement. The first generation luminosity calorimeter (LCAL) covering 45 to $190 \mathrm{mrad}$ with lead-proportional chambers. was sufficient to reach 0.37 to $0.49 \%[2,3]$ and provided a basis for a more precise measurement. The reduction of the beam vacuum-pipe outer diameter from $15.6 \mathrm{~cm}$ to $10.9 \mathrm{~cm}$ for the second year of LEP operation made it possible to replace the small-angle tracker (SATR) modules in front of LCAL, near $\pm 2.5 \mathrm{~m}$ from the interaction point (IP). with very compact luminosity monitors covering the polar angular regions down to $24 \mathrm{mrad}$. Installed in a two week LEP shutdown in September 1992, the SICAL calorimeters gave reliable luminosity measurements from the start. The analysis of the first two month's running is reported in [2]. In this article we describe their design, construction and performance.

\subsection{Design considerations}

At LEP the size of the luminous region is small $\left(\sigma_{x} \simeq 0.14 \mathrm{~mm}, \sigma_{y} \simeq 0.003 \mathrm{~mm}\right.$, and $\sigma_{z} \simeq 7 \mathrm{~mm}$ ) with respect to the scale of the distance to the detectors. thus once the background and detection efficiencies are under control, the limiting source of systematic error in the measurement is given by the error in the acceptance at the lower $\theta$ bound. At small angles the lowest order QED Bhabha cross-section is simply:

$$
\frac{d \sigma}{d \Omega}=\frac{16(\hbar c \alpha)^{2}}{s}\left(\frac{1}{\theta^{4}}\right)
$$

which integrated over the acceptance gives:

$$
\sigma^{2 c c}=\frac{1040 \mathrm{nb} \mathrm{GeV}^{2}}{s}\left(\frac{1}{\theta_{\min }^{2}}-\frac{1}{\theta_{\max }^{2}}\right)
$$

where the polar angular bounds ran be defined as radial limits at a defined z-distance from the IP: $\theta_{m, n} \rightarrow R_{m m}$ and $\theta_{m, 2 x} \rightarrow R_{m, 2 x}$.

The importance of the knowledge of the absolute position of the radial acceptance limit can be sen by convidering the rhange in the measured cross-section due to a displacement of the inner 
radial acceptance bound:

$$
\frac{\delta \sigma^{a c c}}{\sigma^{a c c}}=\frac{2 \delta R}{R_{\min }}\left(1+\frac{R_{\min }^{2}}{R_{\max }^{2}-R_{m i n}^{2}}\right) .
$$

which implies that a desired overall precision of $0.1 \%$ requires control of the absolute value of the radial bound to within $30 \mu \mathrm{m}$ at the distances of $\$ \mathrm{ICAL}$ from the IP. This requirement is fundamental to the mechanical design. Radial resolution of an individual electron's impact may be considerably larger than this value.

The measurement of a scattered $e^{-}$or $e^{+}$in a colliding-beam experiment is potentially compromised by interactions in the beam vacuum-pipe wall. At $28 \mathrm{mrad}$ in ALEPH an electron traverses $5 . \%$ of a radiation length before entering the luminosity monitor. Measurement with an electromagnetic calorimeter solves this problem (see ref. [3]). In such a detector a Bhabha scattering usually deposits two back-to-back showers (radiative Bhabha events may produce three or more showers). Although electrons, positrons and $\gamma$ 's are undistinguishable. their secondary interactions in the beam-pipe produce showers which can be well measured. A calorimeter is sensitive to background coming from the production of two (or three) hard photons $\left(e^{+} e^{-} \rightarrow \gamma \gamma(\gamma)\right)$ ). but the rate of the process is calculable and low $(0.016 \%)$ with respect to Bhabha scattering.

The principal source of background to Bhabha scattering at LEP is the accidental coincidence of off-momentum beam particles. The acoplanarity. of most of these coincidences is an efficient criterium to separate them from the Bhabha events. Furthermore, their energy spectrum within the calorimeter's acceptance is typically $(1992,1993)$ peaked between 20 and $25 \mathrm{GeV}$ when running at the $\mathrm{Z}$ resonance, whereas the mean accepted Bhabha $e^{-}$or $e^{+}$energy is $44.9 \mathrm{Gel}$. An energy resolution better than $40 \% / \sqrt{E}$ is sufficient to separate them from the luminosity events when combined with the acoplanarity rejection.

These considerations, the space available after the removal of the $17 \mathrm{~cm}$ long SATR modules, and the experience in ALEPH with the previous luminosity calorimeter defined the essential design requirements :

1. Full containment of $45 \mathrm{GeV}$ electromagnetic showers requires 24 radiation lengths $\left(X_{0}\right)$. Tungsten as a showering material, with twelve samplings using silicon detectors, gives an average radiation length of $5.2 \mathrm{~mm}$ (compared with $3.5 \mathrm{~mm}$ in pure $\mathrm{W}$ ). This satisfies both space and energy resolution requirements.

2. Precision on the absolute radial fiducial limits of better than $30 \mu \mathrm{m}$ is needed. The well defined pad boundaries of silicon detectors permit this. Using the technique of comparing the energy sharing between adjacent pads at a boundary to define the cuts, this can be achieved for high density samplers, provided the pad to pad response uniformity is better than 1 to $2 \%$.

3. Inderstanding of various systematic effects requires good definition of the shower's radial transverse distribution away from the detector's edges. Radial padwiths near half the Moliere radius $\left(R_{M} \simeq 10 \mathrm{~mm}\right.$ for the material considered) are sufficient for this since typically $90 \%$ of the total energy lies within a cylinder of radius $R_{M}$. Such a pad size is also optimized for energy sharing decisions at the fiducial boundaries.

1. In acoplanarity selection to remove off-momentum background requires azimuthal information. A o-padwidth of $11.25^{\circ}$ with each subsequent laver shifted in azimuth by $1 / 3$ of 
a padwidth $\left(3.75^{\circ}\right)$ is adequate for this purpose. A full $2 \pi$ azimuthal acceptance, without projective cracks, avoids any systematic errors from azimuthal cuts. First order effects from beam-detector offsets can be removed by using asymmetric radial acceptances on either side of the IP and alternating then on a event by event basis.

5. Measurement of trigger efficiency is required for an absolute luminosity determination. Independent triggering on "odd" and "even" layer energy sums gives a cross-check.

\subsection{General layout}

The luminosity monitor consists of two homogeneous cylindrical silicon-tungsten sampling calorimeters which surround the beam pipe at approximately $z= \pm 2.5 \mathrm{~m}$ on either side of the IP. Figure 1 shows a section view of one of the calorimeters. The calorimeters have inner and outer (active) radii of $6.0(6.1)$ and $14.6(14.5) \mathrm{cm}$. respectively: the active volume subtends the polar angular region from 24 to 58 mrad. Altogether twelve layers of tungsten alternate with layers of silicon detectors, described below. Assembly around the beam vacuum-pipe requires that each calorimeter be divided into two halves (right/left). Each half-calorimeter is stacked 11 sing 12 nearly identical minimodules. consisting of an instrumented silicon layer sandwiched between two tungsten plates. described in section 3. The charge amplifiers are described in section 5.1. A motherboard installed at the backplane of each half-module regroups command signals, distributes voltages, and routes the output data. Altogether 12228 channels are equipped for the two full calorimeters. The mechanical precision of the assembled calorimeter is discussed in section 4.

\section{Silicon detectors}

The $1.3 \mathrm{~m}^{2}$ active area required for the two calorimeters is covered using 384 passivated implanted planar silicon (PIPS) $300 \mu m$ thick detectors manufactured by Canberra Semiconductors [6]. The $33.72 \mathrm{~cm}^{2}$ sectors $\left(22.5^{\circ}\right.$ wide) are cut from $10 \mathrm{~cm}$ diameter wafers. shown in Figure 2. They are divided into $2 \times 16$ pads with effective dimensions of $5.225 \mathrm{~mm} \times 11.25^{\circ}$. surrounded by a $100 \mu \mathrm{m}$ wide guard ring. The flatness of the sectors was required to be within $\pm \$ \mu \mathrm{m} / 10 \mathrm{~cm}$. In order to approach the beam-pipe. the inner edge of the sector is cut circularly with a laser, allowing $600 \mu \mathrm{m}$ between the cut and the guard ring. Low fabrication yields $(\simeq 60 \%)$ were associated with this cut.

The PIPS detectors are classical diodes, using high resistivity $(\geq i K \Omega(\mathrm{cm}) n$-type silicon. 'The pads and external guard ring were implanted $\left(\mathrm{B}^{11}\right)$ on the rectifying $p^{+} n$ junction side, where the photolithography assures a pad reproducibility within $\pm 2 \mu \mathrm{m}$. $1100 \mu \mathrm{m}$ inter-pad and guard ring spacing ensures no dead zones when the detector is fully depleted. A 4 .iO $O_{2}$ layer is deposited over the surface. followed by $700 \mathrm{~nm}$ of aluminium over the pads; metallized passages in the $5 \mathrm{SiO}_{2}$ allow $\mathrm{D}$ ( readout of the pads. The noninjecting $n n^{+}$ohmic side ( $\mathrm{P}^{31}$ doped) is overlayed with $300 \mathrm{~mm}$ of aluminium allowing connection of the polarization voltage. The detectors are sufficiently reverse-liased. 1ypically $35 \mathrm{~V}$. to fully deplete the silicon bulk. as shown in Figure 3. Depleted detertor capacitance (ate shown in Figure 4, varies linearly with padsize from $25 \mathrm{pF}^{2}$ to $5.5 \mathrm{pF}$. The dark currents. measured for the final detectors. are lypically less than 5 nd per pad. where production specifications required cark currents less than $20 \mathrm{n:} / \mathrm{p}$ ad and/or less than $100 \mathrm{nat}$ for any is pads on the crestal. 
In order to obtain the thinnest possible silicon detector units, a front-surface readout approach was initally explored. Prototype $300 \mu m$ thick crystals were prepared with DC coupled aluminium rearlont lines deposited over a $5 \mu m$ thick polyamide insulator covering the pads (two companies developed prototypes [7, 6]). The approach was abandoned because of project delays after difficulties with mutable operation due to coupling between read-out lines and the guard ring. cross-talk between pads via the lines, and channel failures due to the fragility of contacts between readout lines and the pads. The adopted solution described below uses printed circuits on a kapton foil bonded to the detector $p$-side.

CESIKA detector sub-assemblies. The individual silicon detectors are mounted on ceramic cupports which position them around the half disk of each mini-module (discussed in the next section). The silicon and ceramic wedges are equipped with $180 \mu m$ thick double-sided printed Kapton readout circuits to form ceramic + silicon + Kapton (CESIKA) sub-assemblies. First. Agloaded conductive epoxy $[8]$ is used to bond the ohmic side of the silicon detector on to the $635 \mu \mathrm{m}$ thick gold-plated cramic support. A ceramic jig. with a similar thermal expansion coefficient. is used to align the silicon detector with the help of a video-microscope. Curing of the assembly requires 2 hours at $120^{\circ} \mathrm{C}$. Optical measurements after curing give a radial precision of $9 \mu \mathrm{mr}$ r.m.s. (consistent with the measurement precision) between a positioning hole in the ceramic and the pads on the crystal. Second, a Ag-loaded epoxy is used to bond the Kapton readout circuit onto the metallized pads, shown in Figure 5. The reliability of this contact was tested on prototypes with accelerated aging methods. The Kapton circuit is attached to the front-end electronics card via a connector. Mechanical and electrical tests are performed before mounting the CESIKA unit.

\section{W-Si-W minimodule construction and calorimeter as- sembly}

Each of the 12 minimodules forming a half-calorimeter sandwich eight silicon detectors between a thin (front) and a thick (rear) tungsten radiator, giving a radiation sampling length of $1.95 \mathrm{X}_{0}$. An additional $W$ plate is bonded onto the front face of the first minimodule so that its sampling thickness is the same. In order to ensure the highest possible detector density, the instrumented gap between the $W$ plates is limited to $2.33 \mathrm{~mm}$, as shown in Figure 6 . Minimodules differ only because of the successive azimuthal offsets $\left(+3.7 .5^{\circ}, 0^{\circ}\right.$, and $\left.-3.75^{\circ}\right)$ used to avoid aligned cracks and to improve the $o$ resolution.

An alloy was used for the tungsten radiators [1]: $97.0 \% \mathrm{~W} .1 .6 \%$ Ni. $0.8 \%$ Fe and $0.6 \%$ Cu. with a resulting density of $18.98 \mathrm{~g} / \mathrm{cm}^{3}$. This choice simplified the machining of the plates to the resired unformity and flatness. The $W$ plates. machined as full disks, are cut in two halves with an angled cut of $36^{\circ}$ with respect to the surface. This ensures that there is no projective discontinuity at the junction of two plates, as shown in Fig. $i$.

The rear 11 plate of a minimodule, $3.8 \mathrm{~mm}$ thick, is bonded to an epoxy-fiberglass " $\mathrm{i}-10$ " plate. which serves both to hold the entire assembly on the support rods as well as to locate the silicon defectors. The dimensional stability of the $\mathbf{i}-10$ plate is critical to the overall precision. The material was chosen for its thermal and hydroscopic properties [5]. Two $16.5 \mathrm{~mm}$ diameter precision holes allow the (i-10 plate to slide onto support rods at the stacking stage. Each ('ESIKA unit seramic support is referenced with a $3 \mathrm{~mm}$ dianeter precision pin (5/m hole tolerance) and held in a sot machined in the plate (edge precision is obtained by moleling). 
The coordinates of all the alignment holes were measured at $20^{\circ}$ ( with a precision of $5 \mu m$ using a computerized scanning table [9]. The values were entered into a database for analysis purposes; the azimuthal position of the holes agreed to within $\pm 0.01^{\circ}$ with the design. Subsequently it was found that the in situ operating temperature is $8^{\circ} \mathrm{C}$ higher (typically $28^{\circ} \mathrm{C} \pm 0.3^{\circ} \mathrm{C} \mathrm{r} . \mathrm{m} . \mathrm{s}$.) than was nued for the measurements of the reference holes. The thermal expansion coefficient of the bonded pair of $\mathrm{G}-10$ and $W$-plates was measured and found to be isotropic. with $\alpha_{T}=(8.0 \pm 0.3) \cdot 10^{-6} /{ }^{\circ} \mathrm{C}$. This gives an increase of the inner diameter of $(9 \pm 3) \mu \mathrm{m}$ with respect to the measurements at $20^{\circ} \mathrm{C}$, including the expansion of the ceramic supports and silicon detectors.

The ('ESIKA detectors overlap at alternate depths around the (- -10 plate: a $0.20 \mathrm{~mm}$ depth difference minimizes azimuthal variations (see sect. 7.2 ). The overlap) with the neighboring silicon detector is centered between the pad edge and the guard ring. The edge of the underlying detector is shadowed by the $1.5 \mathrm{~mm}$ of silicon extending beyond the pad. This shadowing is constant with radius and thus unimportant for the luminosity measurement. Once positioned on the plate, the ('ESIKA units are equipped with the front-end amplifier cards.

The front $W$ plate of a minimodule, $3 \mathrm{~mm}$ thick, is bonded to a thin aluminium plate (machined to $0.5 \mathrm{~mm}$ in the region of the $W$ ) with which it is held on the support rods. Silicon PTC temperature probes are placed on the front aluminium-W plate near selected pre-amplifier chips. This plate is also used to make thermal contact with the water-cooling system. A platinium (PT100) probe is positioned on the central W-plate of each half-calorimeter.

The two plates of the equipped minimodule are screwed together around the edge via the $\mathrm{G}-10$ and aluminium plates.

The calorimeter is assembled by stacking the minimodules together on the $16.5 \mathrm{~mm}$ diameter stainless steel support rods, located $10 \mathrm{~cm}$ from the tungsten outer edge, see Figure 8 . The tolerance of the fit on the rods is $10 \mu \mathrm{m}$. The support rods are maintained parallel by the aluminium calorimeter case, which when closed acts as a Faraday cage to isolate the detector from possible noise generated by other ALEPH sub-detectors. The motherboard and auxilary electronics are mounted on the $8 \mathrm{~mm}$ thick aluminium rear plane of the calorimeter. The last $W$-plate, which is located after the final silicon sampling layer protects the detector from rear entering soft gammas and ensures the same back-scattered component of the shower sampling as seen by the other layers.

The calorimeters are supported on $30 \mathrm{~mm}$ diameter horizontal stainless steel rods fixed to the ALEPH central detector (TPC) support legs, orthogonal to the beam-axis. The silicon detectors are protected against synchrotron radiation coming through the vacuum-pipe wall by a $2 \mathrm{~mm}$ thick lead tube placed around the beam-pipe before closing the modules. The half-calorimeters are then slid into position on either side of the racuum-pipe.

\section{Mechanical stability and precision}

Once installed, a geometrical survey gives the absolute position of each module within $\pm 0.2 \mathrm{~mm}$ in $r$ and $y$. and $\pm 0.5 m m$ in z (along the beam axis). The relative separation between half-calorimeters is measured by 1 gromps of light emitting diodes (LED) and photodionle receivers, giving a precision of sum. calibration uncertainty included. The separations are continously monitored during the cata taking: the maximum drift during a gear's data taking was less than Sfun. Additional LED gronps are installed between the TPC' and the leg supporting the SIC 'AL to monitor movements of the calorimeters along the beam direction, in particular. with the turn-on of the 1.5) Tesla magnetic 
field. The z-positions of both calorimeters over the $5 \mathrm{~m}$ distance are stable with respect to the survey positions within a mesaurement precision of $100 \mu \mathrm{m}$.

Internal mechanical stability depends upon the thermal stability of the detector. The total power dissipation is $70 \mathrm{~W}$ per half-module. Water cooling (temperature stabilized) is sufficient to maintain a constant temperature within $\pm 0.3^{\circ} \mathrm{C}$, thereby keeping the silicon detector radial positions within $\pm 3 \mu \mathrm{m}$.

The various sources of the uncertainty on the silicon detectors absolute mean radius are summarized in Table 1. A total radial uncertainty of $9 \mu \mathrm{m}$ is found $(1 \mathrm{~S} \mu \mathrm{m}$ given in [2] was prior to measurements of $\alpha_{T}$ for $\mathrm{G}-10$ plates, described previously). It assmmes that the errors related to individual silicon detector units. (1), (2) and (3) in Table 1. are reduced by the luminosity measurement in the 16 (ESIKA units in azimuth per layer. The minimodule errors. (5) and (6), are reduced by $1 / \sqrt{2}$, since two layers are used together for the fiducial selection. Finally the overall error is reduced by combining two independent selections using either endcap calorimeter to define a tight fiducial cut. Only the thermal effect is considered to be coherent between both sides.

\section{$5 \quad$ Electronics and readout}

\subsection{Charge amplifiers}

The charges collected from the individual pads of each silicon detector are integrated using a pair of 16-channel "Application Specific Integrated Circuits" (ASIC), each serving half of a crystal in azimuth. The ASIC includes preamplifiers, shaping amplifiers, track-and-hold circuits, a fast-OR for triggering purposes, an internal calibration circuit and digital readout switches. It is based on the CMOS AMPLEX chip developed by CERN for the UA2 experiment's silicon track detector; the present version, adapted for calorimetry purposes and including the trigger and calibration upgrades. is called the AMPLEX-SICAL $[10,11]$. The chip specifications and performance are summarized in Table 2 . Its radiation tolerance, up to about $250 \mathrm{Krad}$. is largely sufficient for use at LEP. A schematic diagram is shown in Figure 9.

The principal modifications in the AMPLEX ASIC which were required for the calorimetry application were: (1) the extension of the dynamic range to $1000 \mathrm{Mip}$ for each channel ("Minimum ionizing particle". where $1 \mathrm{Mip}$ in the $300 \mu \mathrm{m}$ thick silicon detector gives $\simeq 22 \mathrm{~K}^{-}$'s, ref. sect. 7.1). (2) the arddition of a fast-OR (in PRESET mode). where the out put is the analog average of all 16 channels. and (3) the addition of an internal calibration circuit permitting charge injection via $2 \mathrm{pF}$ (externally calibrated) capacitors. with MOS command signals to allow selection of individual channels.

The amplifter response is linear within $1 \%$ over the full dynamic range. ('orrections to the residual non-linearity are made using a parabolic parametrization fit to the output signals. The gain of individual channels increases linearly due to the internal layout from channel 1 to 16 , typically within a full range of $2 \%$. Since the amplifier gain depends upon $C_{t+t}-0.35 \% / p F$. an additional chamnel to channel variation is present due to the radial $C_{t+t}$ dependence of the silicon detectors. The implimentation of the chip was arranged to partially counterbalance these two efferts. The net effect is removed from the data by calibration. The noise also depends upon the 
retector capacitance:

$$
\text { Equivalent . Voise ( Tharge }=800 e^{-}+\left(38 e^{-} / p F \times C_{\text {tet }}(p F)\right) .
$$

Which amounts to $\simeq 0.2$. Mip for the silicon detectors used. This gives an overall noise $<1 \mathrm{ADC}$ count for the complete electronic chain. The output depends upon the HOLD timing, as shown in Figure 10a. The hold timing in the acquisition mode is adjusted so that the maximum pulse height is obtained for the total energy seen in both calorimeters with coincidence triggers, typically $250 \mathrm{~ns}$ after beam crossing. In calibration mode. the same hold timing with respect to charge injection is obtained by maximizing the output signal when injecting $1 \mathrm{~V}$ on two channels ( 3 and 1$)$ of the AMPLEX chip. The analog buffering of the shaped signals ensures a signal drop $<1 . A D C^{\prime}$ count over $10 \mathrm{mss}$. insignificant over the acquisition time scale. A signal on a single channel typically induces a $-1.6 \%$ cross-talk ( $250 \mathrm{~ns}$ HOLD delay) over the other outputs, via the chip substrate. This can be modified by the detector capacitances and adjacent pad coupling. Figure 10b shows the cross-talk for adjacent pads and for a more distant pad on the same crystal. The effect of positive cross-talk for late HOLD values can be seen for full showers summed over many channels from LEP data (shown in Figure 10a). This behavior has been studied in the light of LEP bunch-train operation where beam bunch separations from 200 to $500 \mathrm{~ns}$ are considered.

The A.MPLEX chips are mounted by pairs on front-end cards which are DC coupled via the kapton foil (ref. Figure 5) to the pads of a single crystal; each chip serves the 16 channels of an $11.25^{\circ}$-slice. The card distributes power for the chips (decoupled from the power supplies). the bias volt age for the crystal, and includes a protective $100 \% \Omega$ resistor to measure the reversecurrent. Typical power consumption is $400 \mathrm{~mW}$ per card. An external calibration permitting rapid checkout of the complete chain is included on the card using capacitive coupling between the amplex input signals and a common injection line for 8 inputs. The card receives command signals from the motherboard and uses 4 analog switches to control the charge injection for both internal and external calibration.

The motherboard installed at the back-plane of each half-calorimeter adapts and distributes command signals from the sequencer to all amplex cards via a daughter command-card. and routes the output data to 24-channel daughter data-cards (each serving 12 layers at the same azimuth). Partially shielded flat cables ( 25 pin) connects the front-end cards to the motherboard. where the shielding was added to reduce cross-talk between the AMPLEX command signals and neighboring cards output signals.

The differential output front-end signals are buffered and sent to the electronics barracks via $30 \mathrm{~m} 25$-pair shielded cables. Level adaptors, at the barracks, receive the differential outputs and perform two functions: (1) shift the output levels by $+2 \mathrm{~V}$ to match the input range $(-0.4$ to $4.6 \mathrm{~V})$ of the multiplexed analog to digital converter (MLX-ADC. described in the next section), and (2) send a second output to the trigger electronics.

Common grounding to the experiment is made for data outputs at the MUX-ADC Fastbus rate level. and at the trigger Fastbus crate level for the trigger signals.

\subsection{MUX-ADC's and sequencer}

The analog signals from the front-end electronies are digitized with multiplexed input l'2 bit volt age level digitizers. Fastbus F6815 M“X-ADC'. designed for the ALEPH electromagnet ic calorimeter 
$(E C A L)$. The readout and multiplexing is synchronized with a programmable sequencer, MUX$S E Q$. Each $M I T-A D C$ receives the information from 2 daughter data-cards on the calorimeter's motherboard (described above). corresponding to all 12 layers of four azimuthal consecutive sectors. where the signals from the level adapters arrive via a data board on the auxilary backplane. The full range of the level adapted output signals was chosen large enough such that the ADC' is never saturated for electromagnetic showers from a Bhabha event. The full dynamic range is slightly reduced by the pedestal values: typically $\simeq 300 \mathrm{ADC}$ counts out of 4095 (full range).

Although each MUX-ADC module contains 12 ADC chips processing in parallel, only 6 are used for this application. Inputs to these chips are multiplexed serially by a factor 8 . The individual ADC signal conversion time is $6.875 \mu \mathrm{sec}$, where each AMPLEX provides 16 pad signals and I fast-OR signal to be digitized serially. Altogether the total conversion time. including front-end initialization sequence, takes $\simeq 1 \mathrm{msec}$ for the calorimeter.

A fast hardware "zero suppression" algorithm is implemented in the MUX-ADCs which permits a substantial reduction of the readout volume. Triplets of pad energies (same radial and azimuthal bins in subsequent layers) are compared against their corresponding reference thresholds; all three pads of a triplet are read out if one of them is above 4 ADC counts $(\geq 20 . / 6 V)$. The triplet structure internal to the $\mathrm{MI} X-\mathrm{ADC}$, comes from the original ECAL application. Zero-suppression of the whole calorimeter is performed in $200 \mu \mathrm{sec}$.

The Fastbus F6846 MLX-SEQ module is a programmable secuencer with an $8 . \mathrm{MHz}$ clock used to synchronize the readout and multiplexing of the front-end electronics with the MUX-ADCs. In addition, it sends the command signals in both acquisition and calibration modes, as well as the calibration pulses to the front-end electronics for detector checkout and calibration. It also conforms to the trigger protocol convention used for the various DAQ configurations. An auxiliary sequencer board distributes the ECL differential command signals to the four half-calorimeter module daughter command-cards.

\subsection{Data-acquisition}

In order to ensure a common dead time between the luminosity measurement and the hadronic event collection, and in order to use the added acceptance provided by the calorimeter for $\mathrm{Z}$ decay products (for example) at small angles, the readout is included in the general data-acquisition system as for any of the other sub-detectors. This follows the treelike architecture of the existing instem where the luminosity event information is combined with the information coming from the other sub-detectors into a single event. The event building process accesses the formatted information in the event buffer of the SICAL Event Builder (EB), operating under OS-9.

The detector's DAQ is based on the Fastbus standard. Two Fastbus crates are used: one reads out the front-end electronics and the other implements the trigger decision (described in sect. 6). The readout crate contains 16 F6845 MLX ADC's, one F68.16 MLX Sequencer to drive the ADC's. and an Aleph EB 6858F, used as Fastbus master. In addition. a crate clustering card (C'C) Fos:2E and segment interconnect (SI) are required.

Readout functions are defined as 3 independent tasks: producer. reformatter and consumer. The produce initializes the DAQ (loads the sequencer program and the thresholds into the ADCs). reads the digitizerl zero-suppressed information from the ADC' mordudes and puts the raw data into the event butfer. The procedure is optimized to reduce the event readont time. which, at low trigger 
rates, defines the SICAL dead time, presently limited to about $\tau$ msec.

The reformatter performs the pedestal subtraction of the data and prepares a formatted event consisting of several banks containing calorimeter hits and trigger information to be sent to the rlata stream. The average SICAL event size for Bhabha events (1ypically I20 hits) is 5 Kbytes. raw lata after reformatting.

\section{Trigger}

Three trigger types are required for the luminosity measurement: a double-arm coincidence trigger (SCAL-MED), a very-high threshold single-arm trigger (SCAL-HI) and a very-low threshold single-arm trigger(SCAL-LO). In order to measure the efficiency, each trigger type is formed using redundant, independant triggers derived separately from the odd or even planes by summing the pad energies within $15^{\circ}$ azimuthal segments. Trigger characteristics are summarized in Table 3 . In contrast to the other sub-detectors, the SICAL triggers are fully formed within the sub-detector's own trigger system. The final luminosity trigger logical levels are sent to the general Level-1 trigger (decision within about 5 hsec), thereby respecting the readout protocols.

The double-arm coincidence trigger is required to be $100 \%$ efficient for Bhabha scattering events while giving good rejection of background from accidental coincirlences of off-momentum beam particles. It demands the coincidence of a "localized" high energy deposit, $A_{\text {high }}$ or $B_{\text {high }}$ (typically $\geq 24 \mathrm{GeV}$ ) in endcap calorimeter $A$ or $B$ respectively, with a similar deposit above a less stringent threshold, $B_{\text {low }}$ or $A_{\text {low }}$ (typically $\geq 12 \mathrm{GeV}$ ), in the other endcap. The coincidence, $\left(A_{\text {low }} \cdot B_{\text {high }}\right)+\left(A_{\text {high }} \cdot B_{\text {low }}\right)$, is an OR of four conditions:

$$
\begin{gathered}
\left(\left(A_{\text {low }}^{\text {odd }} \cdot B_{\text {high }}^{\text {odd }}\right)+\left(A_{\text {high }}^{\text {odd }} \cdot B_{\text {low }}^{\text {odd }}\right)\right) \operatorname{OR}\left(\left(A_{\text {low }}^{\text {even }} \cdot B_{\text {high }}^{\text {odd }}\right)+\left(A_{\text {high }}^{\text {even }} \cdot B_{\text {low }}^{\text {odd }}\right)\right) \\
\left(\left(A_{\text {low }}^{\text {even }} \cdot B_{\text {high }}^{\text {even }}\right)+\left(A_{\text {high }}^{\text {even }} \cdot B_{\text {low }}^{\text {even }}\right)\right) \operatorname{OR}\left(\left(A_{\text {low }}^{\text {odd }} \cdot B_{\text {high }}^{\text {even }}\right)+\left(A_{\text {high }}^{\text {odd }} \cdot B_{\text {low }}^{\text {even }}\right)\right) .
\end{gathered}
$$

The very high threshold (typically $\geq 39 \mathrm{GeV}$ ) single-arm trigger is used to cross-check the coincidence trigger efficiency.

The very low threshold single-arm trigger (typically $\geq 9 \mathrm{G} \in V$ ) provides information on the off-momentum beam particle spectrum, used to estimate the contamination to Bhabha scattering. It is substantially downscaled (ref. Table 3) to reduce its impact on the experiment's dead time, allowing enough statistical precision for the background estimate.

Each trigger sector is designed to contain the full energy deposit of an $\epsilon^{-}, \epsilon^{+}$or a $\gamma \cdot$ thus $45^{\circ}$ sectors (octants). corresponding to two silicon crystals. were chosen. In order to properly handle showers overlapping a border between two octants. two separate trigger sector sub-divisions are used: "Roarl 1" and "Road 2", rotated in azimuth by a half-octant with respect to each other, as shown in Figure 11. Since the trigger signals are summed over ODD or EVEX layers, altogether 6.1 sectors are used including both endcaps.

\subsection{Trigger electronics}

The trigger ststem is buile using two dedicated Fastbus rards: the MIXER sum cards, s per endeap. and a single Total sutn rard. The fast-OR analog signals from each endeap are transmitted 
to the trigger system via the level adapters. The MIXER sum cards digitize the signals with flash ADC"s. and then form independent Road 1 and Road 2 digital sums for the odd and even plane sectors as described above. A group of 4 Mixer sum cards forms a summing network to prepare the 16 sector sums for a single endcap and parity (odd/even). The final double-arm and single-arm triggers are constructed on the Total sum card. Field programmable gate arrays (XILINX [12]) are used throughout the system to make and manipulate the digital sums. The final SICAL trigger decisions are sent as ECL signals to the main trigger. The total time needed to make these (lecisions is about 1.5 $\mu \mathrm{sec}$, where all but about $250 \mathrm{nsec}$ is in cable delays.

MIXER sum cards. A block diagram of the mixer, based upon j XILINX gate arrays, is shown in Figure 12. Each card's primary inputs serve either the first or last 3 odd/even layers of four adjacent trigger sectors (Road 1 or Road 2). AC coupled inputs avoids large DC-offsets. The input signals are converted with AC-coupled 7 -bit $(25 \mathrm{MHz})$ flash-ADC's [1:3] (48/card). The digitization timing is defined by a command from the Total sum card derived from the Gated beam crossing. Only the 5 highest bits are used for the sums (the lowest 2 serve for testing). In the case of noisy channels, individual flash-ADC"s may be disconnected via Fastbus.

Once the input signals have been digitized, the Mixer cards form trigger sector sums in two steps in order to share the information from adjacent sectors. First, the four XILINX on each card form intermediate sums with the signals digitized on the card. Then each XILINX forms a total sum for the trigger sector it represents (Road 1 or 2 , odd/even). The remaining offsets measured during pedestal runs are subtracted from the final sum in each XILINX. The total sector sum has a dynamic range of $256 \mathrm{ADC}$ counts, with an overflow bit. A $45 \mathrm{Gel}$ shower typically gives a sector sum of $50 \mathrm{ADC}$ counts.

Finally, the pedestal-subtracted sector sum is compared against the four programmable thresholds described above in the fifth XILINX on the Mixer sum card. The four discriminator masks on a single card are pre-OR'ed and the result is sent to the Total sum card.

Total sum card. The trigger masks from the Mixer sum cards of both endcaps are ORed together in a single XILINX on a Fastbus Total sum card. The 6 basic triggers (ref. Table 3) are generated from the 12 conditions described above and sent to the main trigger, they can also be readout from the card for analysis purposes. At the main trigger these 6 are OR'ed to generate the three SICAL triggers: very low (SCAL-LO), coincidence (SCAL-MED), and very high (SCAL-HI), each of which can be separately downscaled.

The card "handshakes" with the main trigger supervisor in data acquisition mode: all trigger signals remain high untit a level-1 decision (YES/NO) is made, at which point the signals drop. This avoids programming fixed delays into the trigger electronics and guarantees that the Total sum card respects the general protocol.

\subsection{Trigger calibration, rates, and performance}

The trigger energies are calibrated using Bhabha events from the data. The trigger total sums are compared against the $\mathrm{MCX}$-ADC energies read out for the same events, as shown in Figure 13. A linear fit is made for each trigger sector for every LEP fill. The resolution of the trigger electronics is $4 \%$ for the six (odd/even) planes. which is small compared to the calorimeter resolution. The noise of a single sector. due essentially to the frontend electronics. is ypically 1 (iev.

Typical rates for a LEP luminosity of $\left(1 \cdot 10^{31} \mathrm{~cm}^{-2} \mathrm{~s}^{-1}\right)$ are shown in Table 3 . These repend 
both on luminosity and background conditions. Clearly the very high single-arm triggers generally satisfy the coincidence condition, therefore their exlusive rate is typically low $(120 \mathrm{~m} \mathrm{~Hz}$ for the conditions shown). Background studies using the very low single-arm triggers from the 199:3 data sample show a accidental double-arm coincidence contamination of $7 \cdot 10^{-4}$. underlining the low background of the LEP beam at ALEPH during that period.

The coincidence efficiency is shown in Figure 14 versus the energy in the arm opposite to the very high single-arm trigger, where events from Bhabha scattering should satisfy both trigger conditions. The efficiency turn-on is clearly visible above the low threshold at 12 GieV. The coincidence trigger is fully efficient. $100 \%$, above $20 \mathrm{GeV}$. The odd versus even laver trigger efficiencies give very tight limits on their relative inefficiency: $<10^{-4}$. and the overall inelficiency of their OR used in the final trigger is completely neglible.

\section{Performance studies}

Most of the detector's performance requirements for luminosity measurement can be evaluated using Bhabha events collected at LEP; this was important since the tight construction schedule only allowed for prototype tests of critical performance aspects with at most only a partially complete detector. We describe here the test beam measurements of the peak charge collected on the final design silicon pads as a function of shower depth and incident beam energies, necessary for the AMPLEX redesign. The linearity of the amplifier over the range covered, ensures the calorimeter's linearity to sufficient precision. Test beam studies of shower response as a function of air-gap separation at the silicon layers give some insight into azimuthal variations observed in the LEP running. The essential measurements, performed with a final detector prototype and a silicon-strip tracker, to determine the energy sharing asymmetry at the pad boundary are described, and compared with LEP data. Full shower developement in the detector is shown using LEP data. The detector's uniformity and stability are shown using the data collected in 1992 and 1993 at LEP.

Concerning the operation at LEP, it should be remarked that the detector has shown no clear sign of radiation damage in the two and a half year's running. No loss of channels has occured during the rumning periods. Furthermore, noise levels, as indicated by pedestal studies, are stable over two years' running. Clearly, estimated radiation levels at the detector faces have been low, $\leq 300 \mathrm{rad} / y \in a \mathrm{r}$. Two AMPLEX cards out of the 384 have occasionally become noisy, typically cured by cycling the low voltage. The first appearance of the effect. in coincidence with a beam loss, lead to a conservative running procedure. whereby the silicon detector reverse bias voltages are set to zero during LEP beam injection and adjustments. The front-end amplifier voltages are kept on to ensure the detector's temperature stability.

\subsection{Single pad MIP and maximum response}

In order to define the design parameters for the calorimeter version of the AMPLEX ASIC (ref. sect. 5.1) measurements were made of the largest single pad response to clectron showers. A tungsten prototype with a single silicon detector was nsed, reading ont only one pad with the beam centered on it. The shower profiles were determined by varying the thickness of tungsten in front of the silicon, with $\geq 1.5 \mathrm{X}_{0}$ behind to keep backscattering effects constant. A scintillator 
telescope defined the incident particles. and a $1 \mathrm{~m}$ thick iron absorber followed by a counter tagged muons. Measurements with $70 \mathrm{GeV}$ muons defined the energy deposit for MIP: the most probable cnergy deposit in the $300 \mu m$ thick fully depleted silicon detectors gives $1 . \mathrm{MIP} \equiv 3.6 \mathrm{fC}$. Electron beams of 35. 50. 70 and 100 GeV were used to study the longitudinal profile. Figure 1.5 shows the comparison beewen the test beam results in a 50 (iel electron heam and data taken at LEP with the final detector. Figure 1.5a shows the energy distribution at $6.5 \mathrm{X}_{0}$. As an estimator, a Gaussian was fitted to the peak of the response curve. The most probable value is shown versus shower depth in figure $1.5 \mathrm{~b}$. The continuous curve is the best fit to the test beam data with a function of the form:

$$
Q=q 0 t^{2} c^{-h t}
$$

where $t$ is the thickness in $Y_{0}$. The points are the results for Bhabha erents at LEP. The design limit for the AMPLEX was determined using the 100 GeV data: the peak charge on a single pad at shower maximum was $2250 \mathrm{fC}$. A limit charge of $3.8 \mathrm{pC}$ was conservatively chosen to allow for shower fluctuations, and variation of pad size.

\subsection{Single pad response versus $S i$ - $W$ separation}

The silicon detectors in the final detector are alternately overlapping in azimuth. In order to investigate the sensitivity of the response to variations of the air gap between the layer and the tungsten in front of it, a study was made using a single detector element placed behind $5 x_{0}$ of tungsten. followed by $i X_{0}$. The results for an incident $50 \mathrm{GeV}$ electron beam are shown in Fig. 16. showing a $-.5 \% / \mathrm{mm}$ loss.

In addition to the absorption of soft electrons in the air gap, reducing the signal in the silicon as the space increases, the absorption of backscattered shower constituents from the following tungsten plate is also expected in the G-10 plate supporting the detectors [14]. Since the support plate thickness is staggered in azimuth to permit the overlap of the detectors, the two effects are opposed. A $4 \%$ shift of the total energy between alternate silicon detectors, observed at LEP, is assumed to result from the combined effect.

\subsection{Radial transverse energy distribution}

Shower containment defines the detector's useful fiducial volume. The radial transverse distribution for a single azimuthal padwidth at a depth of $8 X_{0}$ (shower maximum) is shown in Figure 17 for LEP data centered (a) on a pad boundary. (b) on a pad center. Essentially full containment $(\geq 9.5 \%$ of the energy) extends from the third radial bin from the calorimeter edge. consistent with the expected $R_{y}$. A typical t5 (iel electromagnetic shower within this volume deposits its energy over the full depth in 80 to 100 pads $\left(E_{p a t} \geq 10.1 / \mathrm{C}\right)$ depending inversely upon the incident radius.

\subsection{Radial energy asymmetry versus depth}

Radial fiducial elections of events in the calorimetere essential to the luminouity measurement. are based upon radial energy sharing between adjacent pads. The radial energy asymmetry 1 , is 
defined by:

$$
A_{r}=\frac{E_{i n}-E_{\text {out }}}{E_{\text {in }}+E_{\text {ont }}}
$$

where $E_{m}$ is the energy inside a radial padwidth within the fiducial region and $E_{\text {out }}$ is the energy in the adjacent padwidth outside the region. Figure 18 shows the ypical 1 , variation with radius near the pad boundary. The luminosity systematic uncertainty $\delta \mathcal{L} / \mathcal{L}$ depends upon the radial dependence of this asymmetry at the fiducial boundary. and is given by the formula:

$$
\frac{\delta \mathcal{L}}{\mathcal{L}}=\frac{2 f}{R_{\min }}\left(\left(d . A_{r} / d r\right)^{-1} \delta . A_{r}\right)
$$

where $f$ is a geometrical factor depending upon the inner and outer fiducial cut radii $(f=1.24$ for the Bhabha selection outlined in sect. 7.7$)$ and $\delta A_{r}=1 / 2(\delta E / E)$, the cell-to-cell energy uncertainty.

A second test beam prototype, with a final design silicon sampling laver (minimodule. ref. sect. 3) was used to measure the $d . A_{r} / d r$ slope at the pad boundary as a function of shower depth. The measurements were performed for four silicon sampling layers, with an incident 50 GeV electron beam. The incident electron trajectory was defined using $16 \times 20 \mathrm{~cm}^{2}$ silicon strip detectors (2 $\mathrm{mm}$ strips) adapted from the NA14 silicon active target [15]. positioned $30 \mathrm{~cm}$ upstream of the prototype. The strip detectors were arranged as a telescope, two groups for $x$ and two for $y$. with staggered planes such that the effective resolution for a track in four $x$ or $y$ planes was $(500 / \sqrt{12}) \mu m$. A scintillator telescope defined the latch timing. and a veto counter was placed $40 \mathrm{X}_{0}$ behind the prototype to remove muons. The beam spot at the calorimeter face was $12 \mathrm{~mm}$ r.m.s. in $x$ and $y$, which accepted tracks crossing two pad boundaries of the prototype at the same measurement point. The precision of the pad geometry was used to cross check the extrapolated track position.

The results for fully contained showers near the boundary between radial pads 4 and 5 are shown in Fig. 19, where the inverse of the fitted slope, $\left(d A_{r} / d r\right)^{-1}$. at the pad radial boundary is plotted versus shower depth for single pads. The increase of shower width causes $\left(d A_{r} / d r\right)^{-1}$ to increase with depth as expected.

Also shown on Figure 19 are asymmetry slopes for LEP data. Since there is no tracking detector in front of the luminosity calorimeter at LEP. we have used the "non-projective" geometry of the detector to constrain the extrapolated shower centroid. Energetic showers (mean energy of 44.9 ( $\mathrm{ieV}$ ) are selected if the centroid in one of the adjacent layers is near the radial pad boundary (pads 4 and 5). obtained by requiring that $A_{r} \rightarrow 0$ for that layer. The extrapolated centroid is determined from this position and the beam IP. using the known geometry of the layers. The asymmetries in adjacent lavers for the same showers are fitted against each other using the extrapolated position information to extract the slope versus layer shown in Figure 19. Small radial corrections $(\mathrm{O}(10$ to $25 \mathrm{fm})$ ) due to pad curvature are not taken into account here since only the differences in these between lavers enters in the extrapolations. The $(d d / d r)^{-1}$ slope in the first layer $\left(2 X_{0}\right)$ is wider for the LEP data compared to the test beam; an EGS simulation including the ALEPH solenoid's 1.5 Tesla B-field shows the same effect. due to preshowering in the beam pipe i0 to s) com upstram.

This stuly thows that an acceptance cut based upon asymmery selections from samplings at depths of 6 to $8 X_{0}$ would have a radial precision of $\leq 20 \mu m$ for a single pair of pads if the

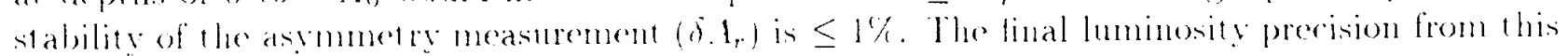
dispersion will be reduced by the large number of measurements in azimuth ( $2 \times 32$ pads). 


\subsection{Longitudinal profile and upstream material}

The advantages of fine segmentation of shower information can be seen in the calorimeter's use to understand the distribution of upstream material. Entering particles must first traverse the aluminum beam-pipe at small angles (2: to 58 mrad) and then pass through a background monitor $(S A M B A \simeq 2 \% \mathrm{Xo})$. The total thickness traversed varies smoothly from $5.4 \%$. Ko at $28 \mathrm{mrad}$ (inner fiducial cut) (lown to $34 \%$. Xo at $48 \mathrm{mrad}$ (outer fiducal cut), excepting locally for two beam-pipe support rings ( $\simeq 12 \%$ Xo). The Bhabha event longitudinal shower profile shown in Figure 20a is used to study the variation of material with incident angle.

Figure 20b shows the relative mean shower depth versus polar angle for both the A-side and B-side calorimeters. The increase with angle agrees well with the measured beam racumm-pipe wall thickness. A beam-pipe support ring $\left(0.12 \mathrm{X}_{0}\right.$ thick) can be seen only on the B-side. since it was permanently removed on the A-side to allow installation of the silicon vertex detector.

\subsection{Energy uniformity and resolution}

The uncalibrated detector uniformity is $\simeq 3.5 \%$ most of which is due to the dispersions of the AMPLEX chips (re. sect. 5.1), found to be stable over a year's ruming. Periodically the response of each AMPLEX is measured by individually pulsing all channels. The average gain (parabolic fit over 5 injected levels) and relative interchannel slope per chip are sufficient to yield residuals below $0.7 \%$. thereby reducing the number of constants for the 12288 channels from 36864 to 38.56 .

Applying the calibration constants, and accounting for the observed $4 \%$ shifts in alternate silicon detector response due to the different air-gap and support thicknesses, yields an energy resolution of $4.9 \%$ for fully contained $45 \mathrm{GeV}$ electromagnetic showers (LEP data); $\sigma_{E} / E=$ $34 \% / \sqrt{E}$.

\subsection{Luminosity measurement}

A description of the luminosity analysis of the 1992 data and the systematic uncertainty in the measurement is given elsewhere [2]; in this section the selection procedure is outlined and used to show the detector's efficiency to remove the off-momentum background. The stability of the luminosity measurement is shown in a comparison with the number of hadronic $Z$ decays collected by ALEPH during the same runs.

The Bhabha scatterings (typical event shown in Figure 21) are selected from luminosity coincidence triggered events by requiring at least one reconstructed cluster in each endcap with an energy $\geq 14 \%$ of the beam energy $(\geq 20$ ( iel at the $Z$ peak). Their stm must exced $60 \%$ of $\sqrt{s}(\geq 5$ (iel at the peak) to remove the off-momentum beam background from the Bhabha scatterings. Figure 22 shows the typically clean separation obtained between the Bhabha peak and the off-momentum background. Radial fiducial cuts (one cluster within a "tight-acceptance". and the opposite within a "loose-acceptance") based upon the energy sharing asymmetry described previously: are nsed to refine the accepted cross-section. The tight and loose cuts are alternated for rach erent te reduce the sensitivity to displacenents of the luminous region. An acoplanarity

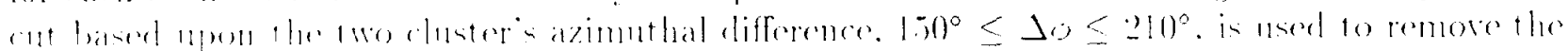
remaining off-momentum beam background without severly cutting away the radiative Bhabha 
events. Figure 2:3 shows the typical distribution with the low background conditions in 1993 .

$A$ cross-check of the stability of the luminosity selection can be seen in Figure 24 where the ratio of Bhabhas (background subtracted) to TP(-selected hadrons is plotted versus fill number. The ratio is constant within the statistical precision of the points.

\section{Conclusions}

Since its installation in September 1992, the silicon-tungsten calorimeter has shown itself well adapted to the goals fixed for the luminosity measurement; analysis of $0.74 \times 10^{6}$ Bhabha events collected during the two month running period in 1992 gave an estimated experimental precision of $0.15 \%$ [2]. limited by the statistics of the Monte Carlo simulation. Considerably more data collected in 1993, $6.4 \times 10^{6}$ luminosity events. has confimed the reliability of the detector and allowed more refined systematic studies, which should give an experimental precision $\leq 0.1 \%$. The ultimate limit of the absolute luminosity measurement is defined by the knowledge of the absolute radial position of the silicon pads used for the fiducial selection and the absolute distance along the beam-axis between the two calorimeters. yielding somewhat better than $5 \times 10^{-4}$ for the detector described here. This is substantially better than the current theoretical $0.25 \%$ uncertainty of the Bhabha scattering cross-section evaluated by Monte Carlo generators [16]. although efforts are underway to reduce this error $[17]$ to between 0.09 and $0.16 \%$.

\section{Acknowledgements}

We express our appreciation to Jack Steinberger for his interest in this project from its start, and thank Jacques Lefrançois for many critical discussions and encouragement. We are grateful for the support given by the ALEPH collaboration, and acknowledge the efforts of D. Hansen and the LCAL luminosity group from whom the SICAL design and analysis benefited.

We particularly acknowledge the effort of the technical teams who have worked to build the detector at ('E-Saclay, to the CERN teams responsable for the production of the AMPLEXSICAL chip. front-end cards, level adapters and the trigger system, and to the Pisa team who worked on the calibration and slow control system. We thank P. Burger and the engineers at Canberra who produced the silicon detectors. and M.O. Lampert and those at Intertechnique for their help in providing silicon detectors during the prototype studies. Finally we thank our laboratories: D.AP.VIA/CE-Saclay (Direction des Sciences de la Natiere. ('E.A.). (ERN and INFN Pisa (Dipartmento di Fisica dell' 'nversità. e Scuola Normale Superiore). for the continued support which has made the SICAL construction possible.

\section{References}

(1) D. Decamp ot al. Vinel Instru. and . Heth. A294 (1990) 121.

[2] D). Buskinlir of al. Z. Phy. C 62 (1991) 5399.

[3] D. Deramp of al. Z. Phy. C 53 (1992) 375. 
[1] Tungsten D185, Plansee, Reutte. Austria.

[5] ME730 epoxy-fiberglass Societe Permali. Maxeville. France. The linear thermal expansion coefficient $\sigma_{T}$ is $1.3 \cdot 10^{-5} / \mathrm{C}^{\mathrm{C}}$.

[6] Canberra Semiconductors X.V.. Olen. Belgium.

[7] Eurisys Veasurements (previously Enertec-Intertechnique). Lingolsheim. France.

[8] Epotecny: Pontoise. France.

[9] S.T.('. Socient. Malakoff. France.

[10] E. Beuville et al., IEEE Trans. Vuclear Science 39 (1992) 766:

E. Beuville et al.. Nucl. Instrum. and .Methods A288 (1990) 157.

[11] MEITEC'N.V., Oudenaarde. Belgium.

[12] XILIXX field programmable gate array, model XC 3064 (70 .HHz toggling frequnecy).

[13] Motorola $i$ bit. $25 \mathrm{MHz}$ converter. MC 10321.

[1.4] H. Hirayama. Proc. IEEE Vuct. Sci. Symposium. Orlando. Fla. (1992) 25-t.

[15] R. Barate et al. Nucl. Instrum. and Methods A235 (1985) 235.

[16] W. Beenakker and B. Pietrzyk, Phys. Lett. B304 (1993) 366.

[17] B.F.L. Ward, S. Jadach, E. Richter-Wạs and Z. Wạs, "Precision Calculation of the Small Angle Bhabha Cross Section", 1994 Rochester Conf., Glasgou Prfcedings (1994). 
(1) Silicon pad relative to (ESIKA alignment hole $9 \mu \mathrm{m}$

(2) Alignment pin, CESIKA and G10 hole tolerances $5 \mu \mathrm{m}$

(3) Alignment hole position on G10 support $5 \mu \mathrm{m}$

(4) G10 support thermal distortion effects $\quad 3 \mu \mathrm{m}(16 \mu \mathrm{m})$

(5) Support rod and hole tolerance $10 \mathrm{~mm}$

(6) Support rod hole position on G10 support $5 \mu \mathrm{m}$

(i) Half-calorimeter separation LED precisions i $\mathrm{km}$

(8) LED sensors calibration uncertainty \& $\mathrm{mm}$

Table 1: Summary of silicon detector absolute radial uncertainties. Values in parenthesis ( ) were used in the 1992 luminosity analysis [2]. Total uncertainty is defined with respect to the final fiducial selection.

Chip technology

Number of input channels

Full dynamic range

Equivalent noise charge $\left(C_{\text {det }}=50 \mathrm{pF}\right)$

Output voltage swing

Maximum allowed input current

Peaking time

Linearity

Resolution

Gain spread

('ross talk (depends upon HOLD timing)

('ommand signals (EC:L)

Fast-OR linearity range

Internal calibration capacitors

Power consumption

\section{$3 \mu m$ CMOS}

16

$3.8 \mathrm{pC}(1000 \mathrm{MIP})$

$0.5 \mathrm{fC}(0.13 \mathrm{MIP})$

$5.6 \mathrm{~V}$

$400 \mathrm{nA} /$ channel

$250 \mathrm{~ns}$

$1 \%$ (full range)

\pm 1 MIP

$\pm 1 \%$

-1.6 to $-2.2 \%$

CLOCK, PRESET. (HANNEL SELEC'T

500 MIP

$2 \mathrm{pF}( \pm 0.3 \%$ within chip)

$200 \mathrm{mll} / \mathrm{Chip}$

Table 2: Specifications of the AMPLEX-SI 'AL multiplexed charge amplifier. 


\begin{tabular}{|c|c|c|c|c|}
\hline Trigger & Description & Threshold(s) & $\begin{array}{l}\text { Down scale } \\
\text { factor }\end{array}$ & $\begin{array}{l}\text { Rate } \\
\mathcal{L}=10^{31}\end{array}$ \\
\hline $\begin{array}{l}\left(A_{\text {high }} \cdot B_{\text {low }}\right) \\
\left(A_{\text {low }} \cdot B_{\text {high }}\right)\end{array}$ & $\begin{array}{c}\text { Double-arm } \\
\text { (Bhabha coincidence) }\end{array}$ & $\begin{array}{l}\geq 24 \text { GeV (high) } \\
\geq 12 \text { GeV (low) }\end{array}$ & 1 & $2 \mathrm{~Hz}$ \\
\hline $\begin{array}{l}A_{v \in r y h i g h} \\
B_{\text {very high }}\end{array}$ & $\begin{array}{l}\text { Very high single-arm } \\
\text { (trigger efficiency) }\end{array}$ & $\geq 39 \mathrm{GeV}$ (very high) & 2 & $1 \mathrm{~Hz}$ \\
\hline $\begin{array}{l}\text { Avery low } \\
B_{\text {very low }}\end{array}$ & $\begin{array}{l}\text { Very low single-arm } \\
\text { (beam background) }\end{array}$ & $\geq 9 \mathrm{GeV}($ very low $)$ & 2500 & $50 \mathrm{mHz}$ \\
\hline
\end{tabular}

Table 3: Luminosity triggers. Inclusive rates (downscaled) are given for a typical LEP luminosity of $10^{31} \mathrm{~cm}^{-2} \mathrm{~s}^{-1}$. Typical very high single-arm exclusive rate is $120 \mathrm{mHz}$. 


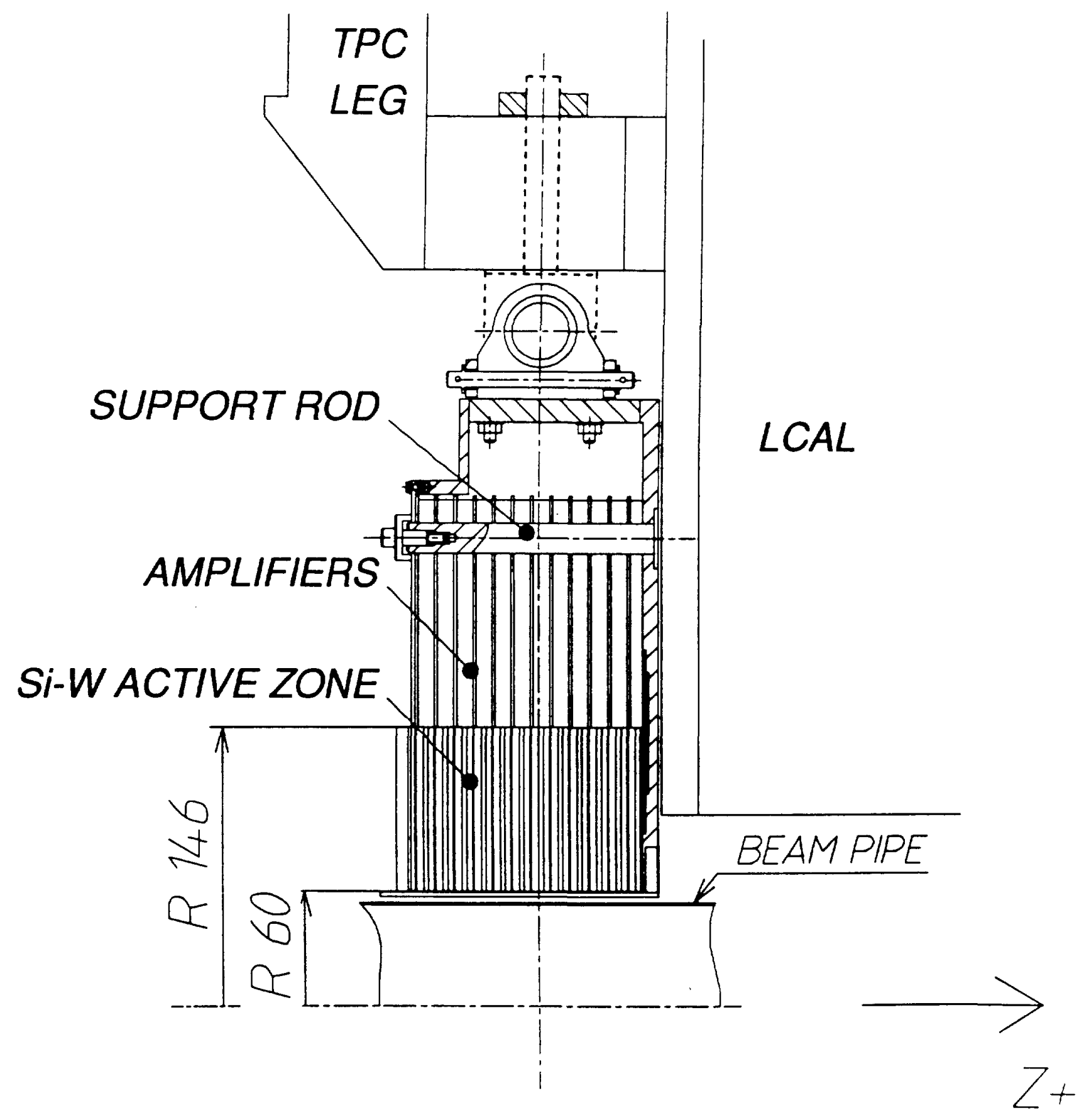

Figure 1: Siection view of one SIC'AL calorimeter. The twelve sampling layers are formed from IV-Si-W minimodules which are supported internally on rods passing through G-10 and aluminium support plates. 


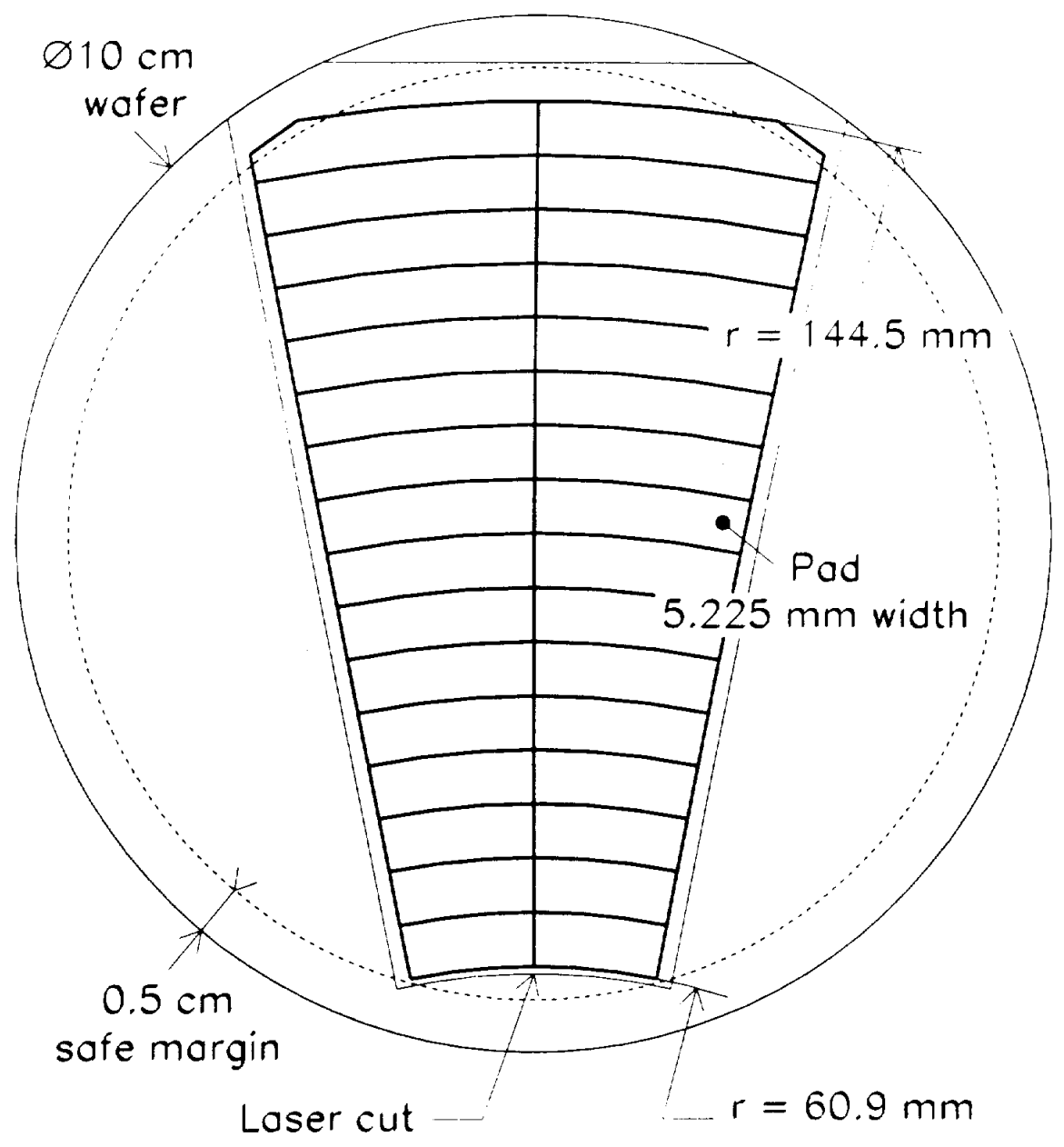

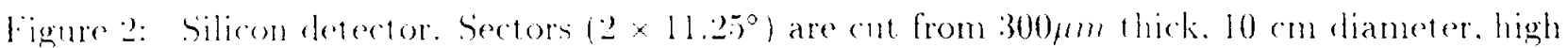
resistivity wafers. The rounded lower radial edge is laser cut. Radial padwidth is $5.2 .5 \mathrm{~mm}$. 


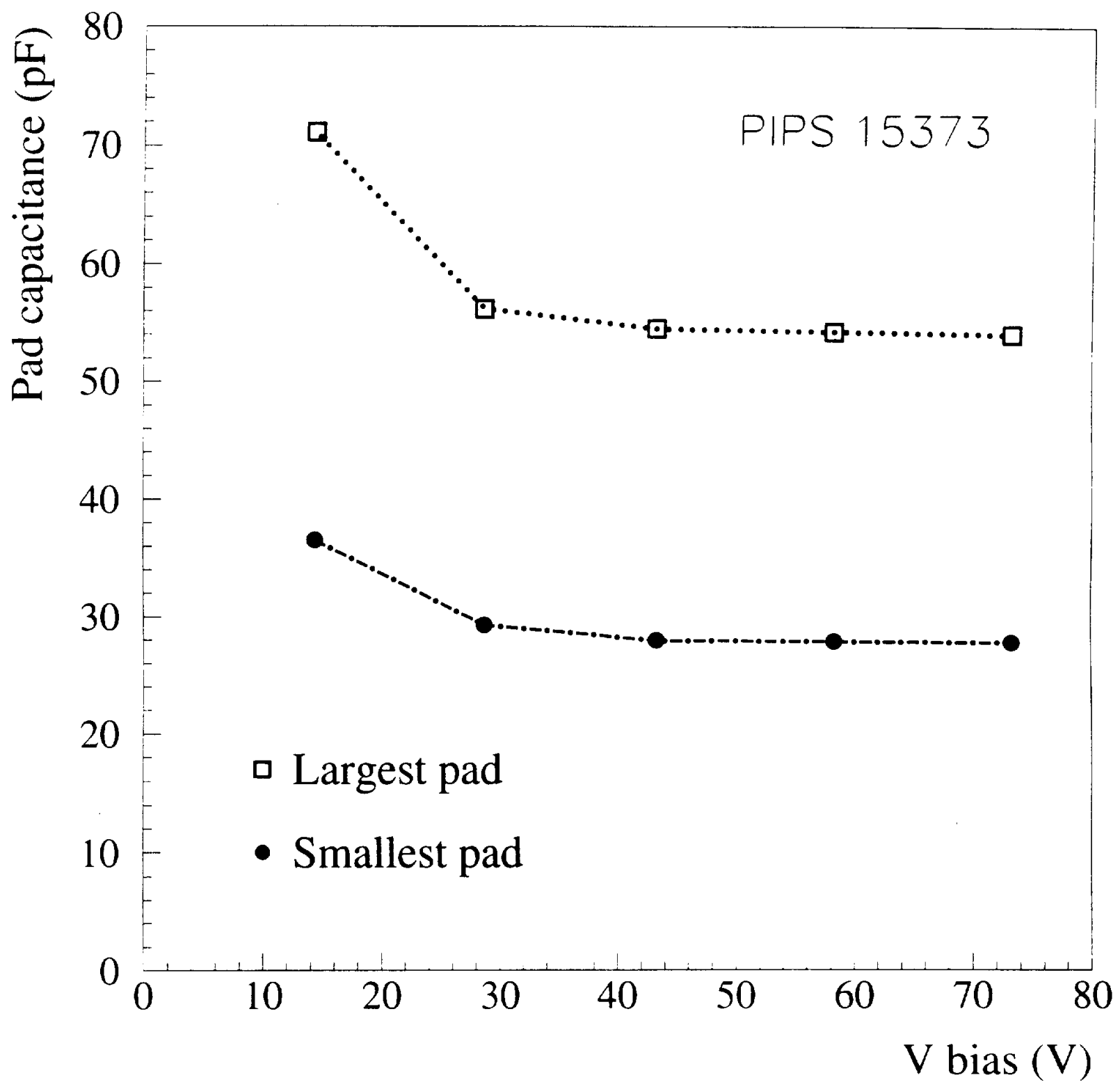

Figure 3: Depletion curves for the largest (open squares) and smallest pads (full circles) of a trpical silien revector. showing full depletion at $3.5 \mathrm{l}$. 




Figure 1: Silicon detector raparitance of a fully depleted eryetal varies linearly from 25 pF (inner

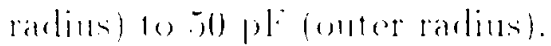




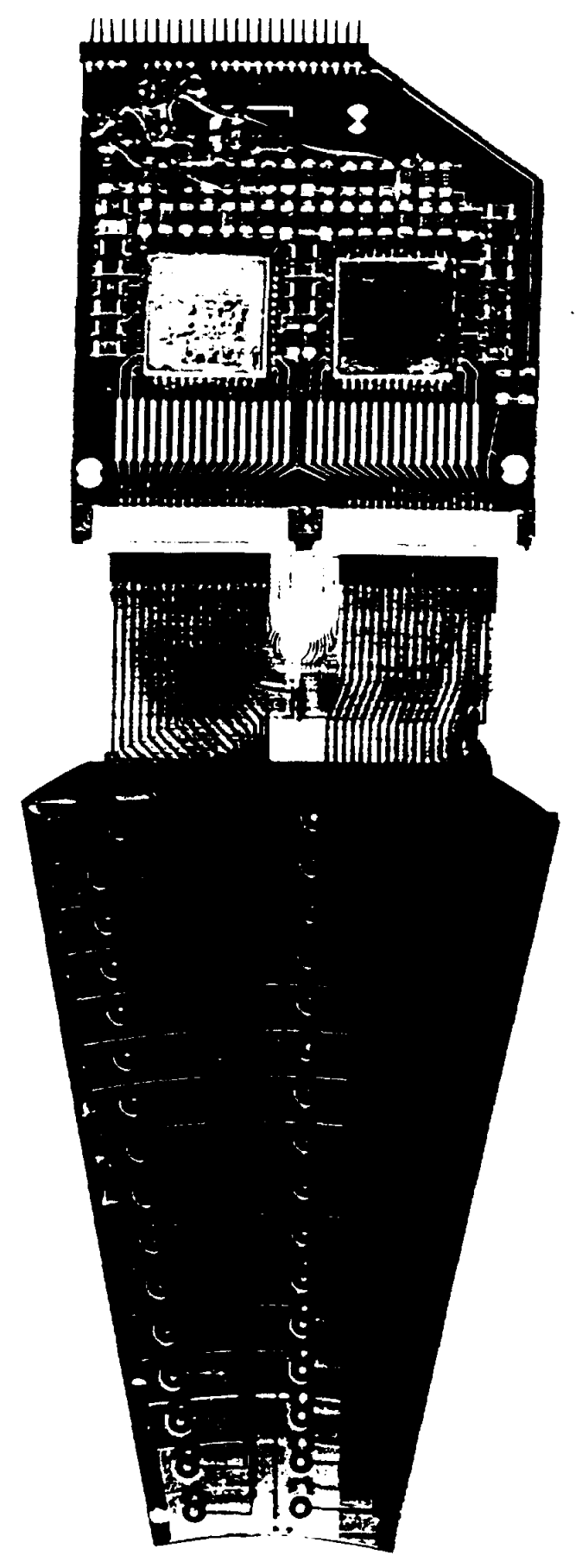

Figure 5: Silicon detector mounted on its ceramic support (cLSIKA assembly). Two charge amplitiors each serse 16 radial pard rows in a single $11.25^{\circ}$ azimnthal slice. Connection to the pads is obtainerl via printed kapton foil. 


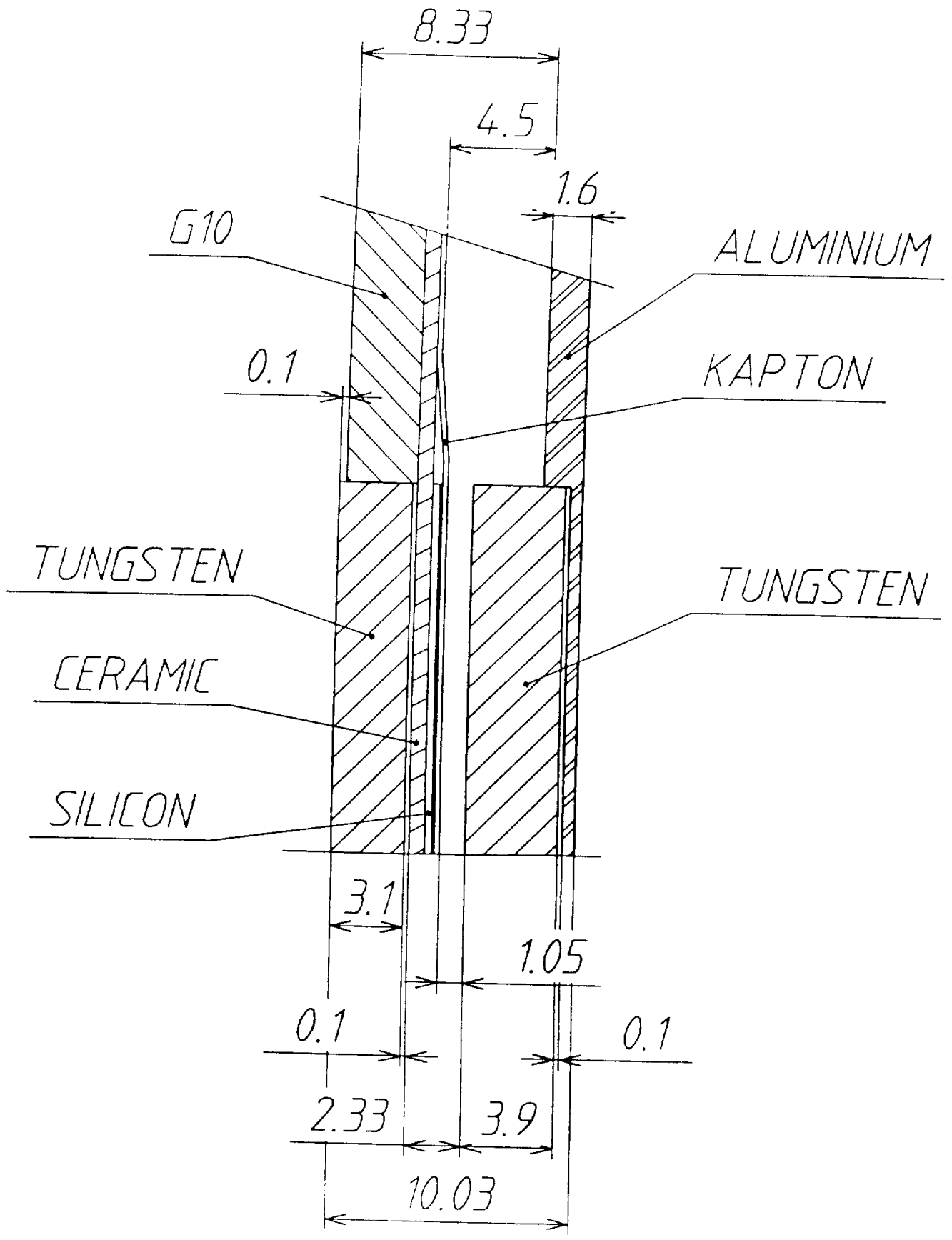

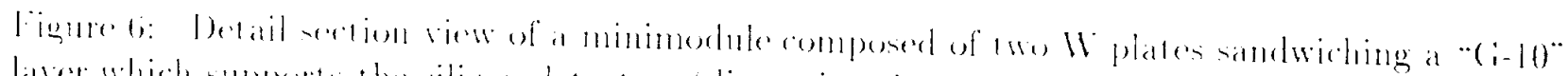

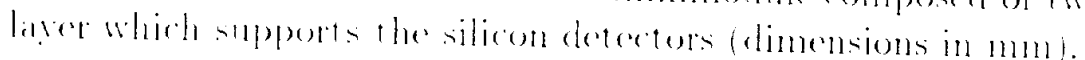




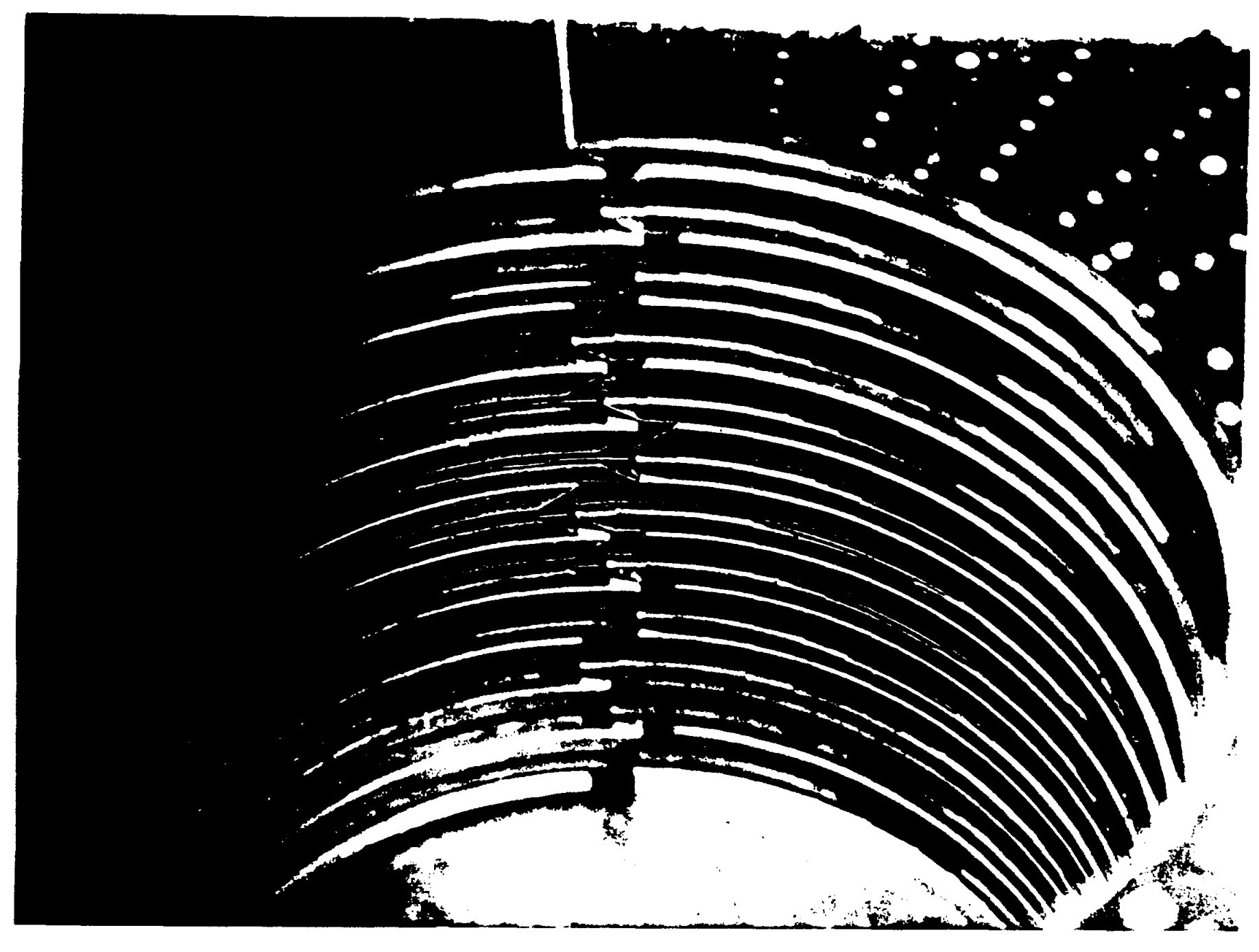

Figure i: Detail photograph of the staggered crack between two half-calorimeters, taken during an isumble test prior to denector completion. The angled ont of the tungsten plates is clearly visible. 


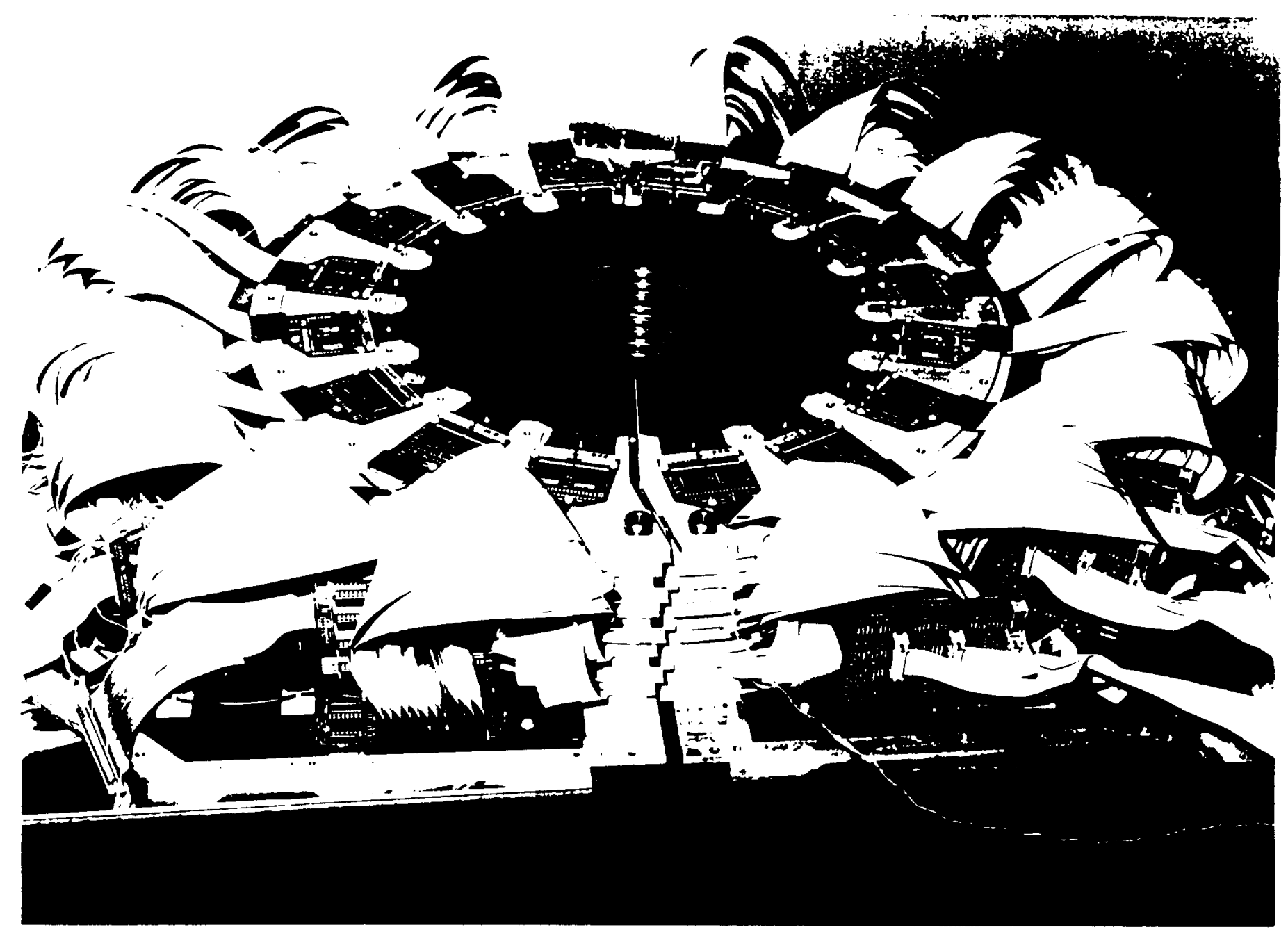

Figures: Staking of calorimeter minimodules. The support rod position each minimodule to a

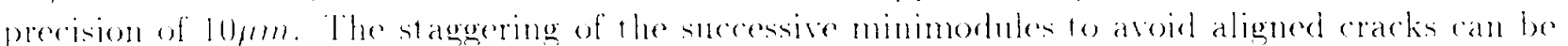
reen in this pheteglaph. 


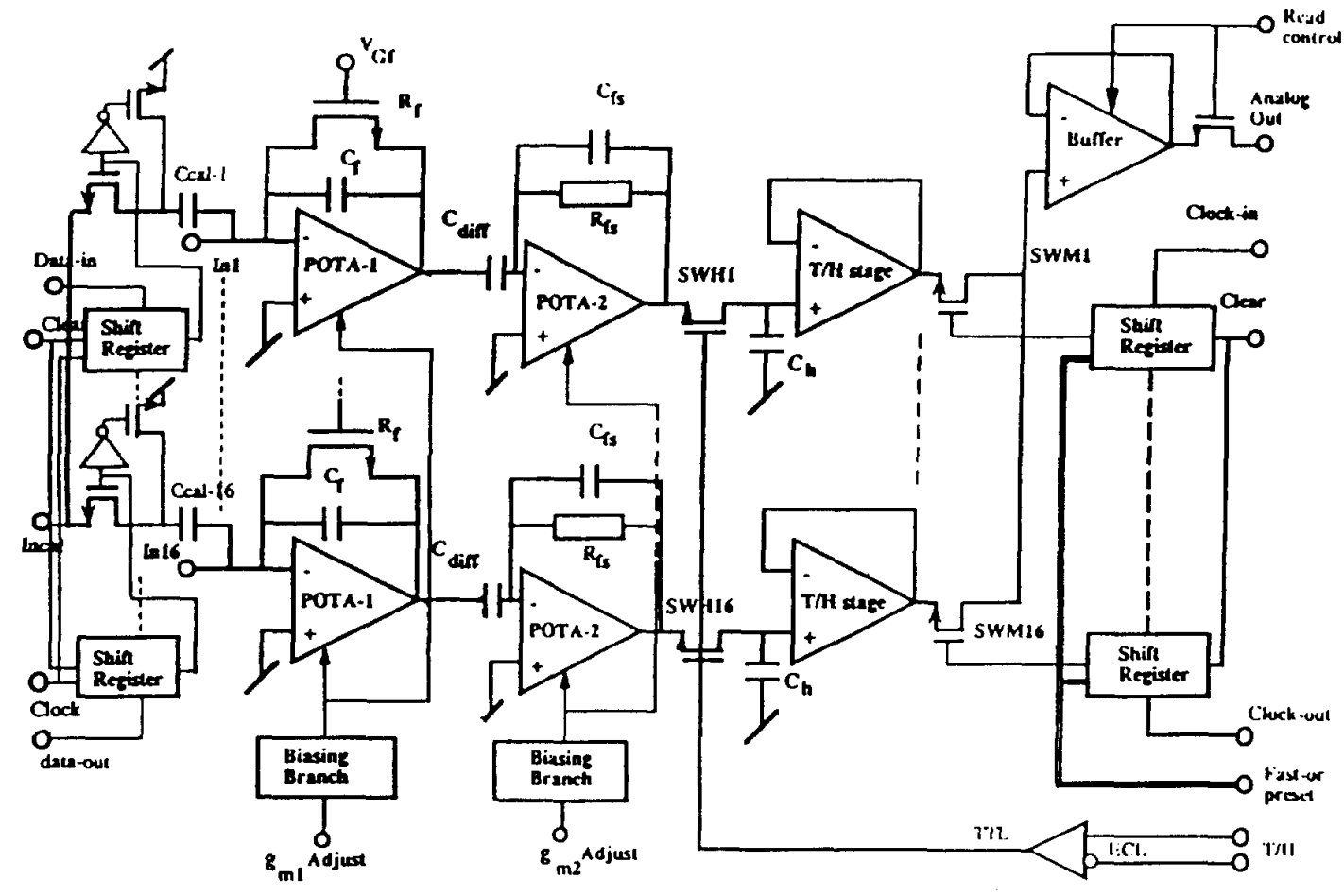

Figure 9: AMPLEX-SIC AL sehematic diagram. 


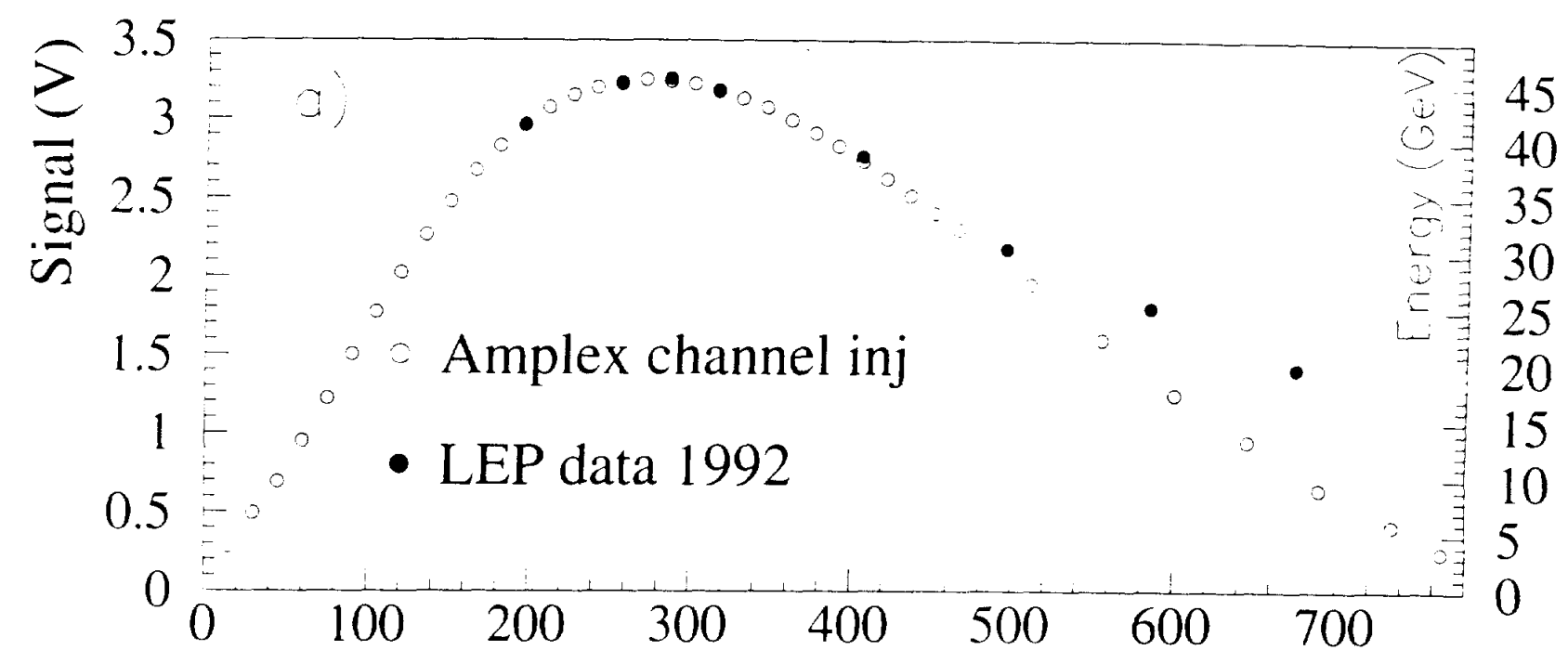

Hold delay (ns)

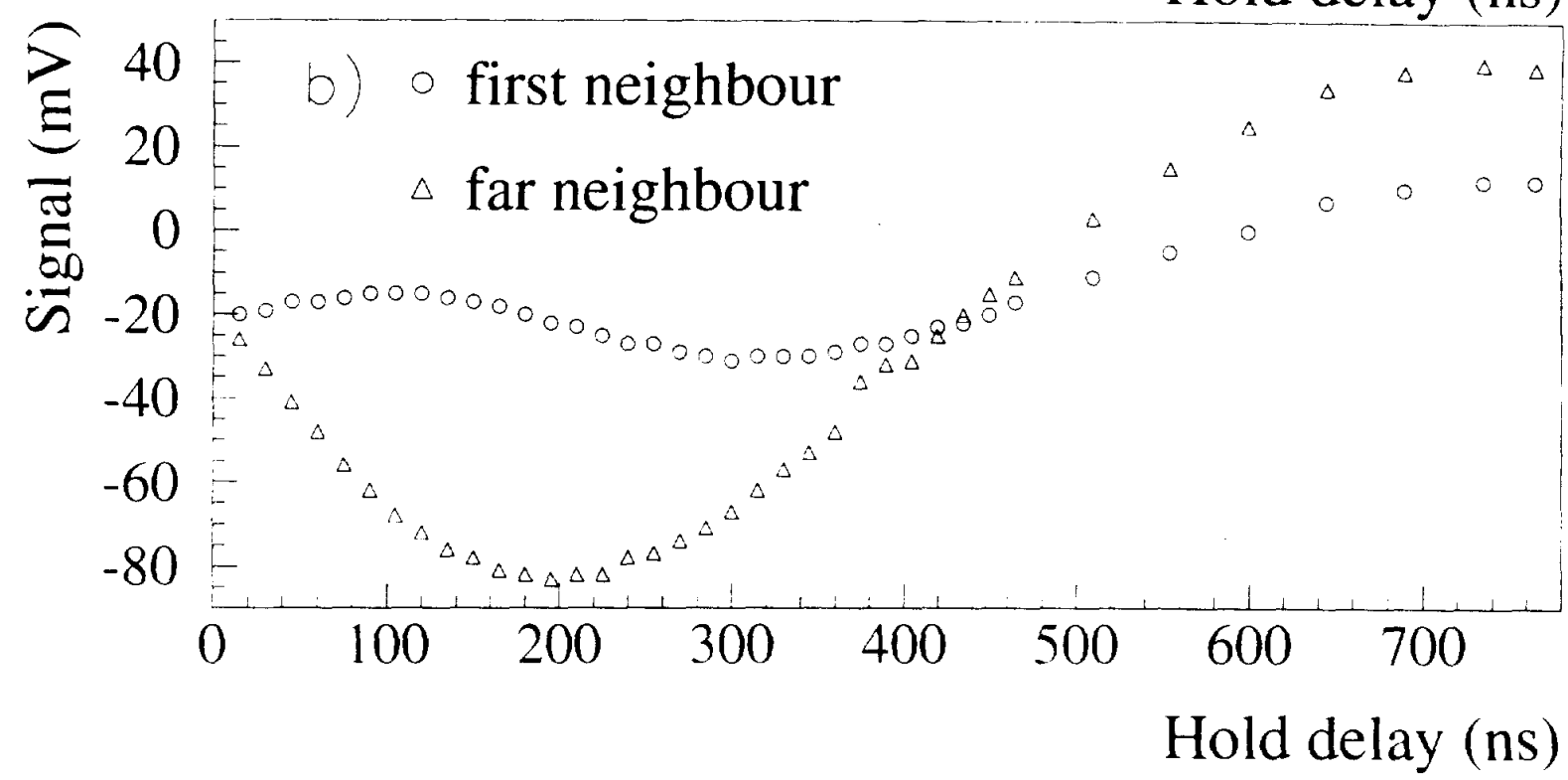

Figure 10: (a) Single channel output (V) versus HOLD delas. ('ross-talk effects are seen when stmming over showers (LEP rlata in full circles) taken at different HOLD settings. (b) ('ross-talk

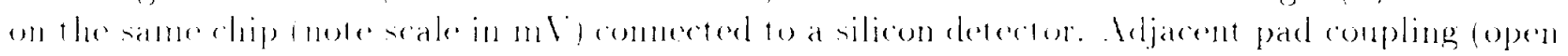

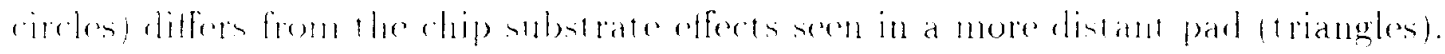




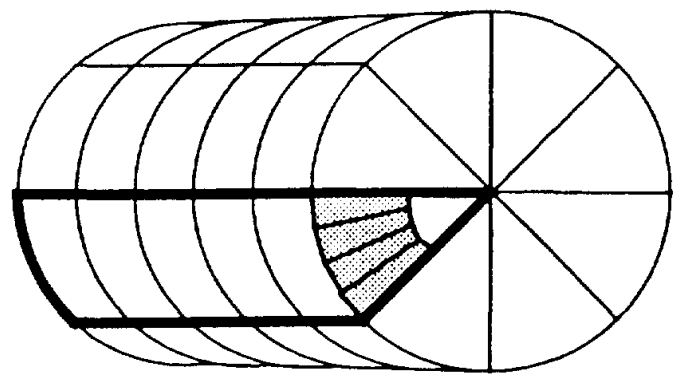

"Road 1"

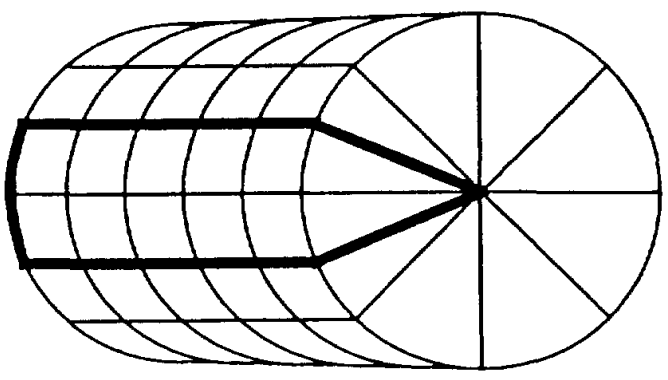

"Road 2"

Figure 11: Trigger segmentation: each trigger sector is $45^{\circ}$ wide. Two sub-divisions. "Road 1"

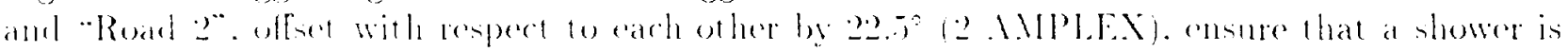
fully contained in at least one. 


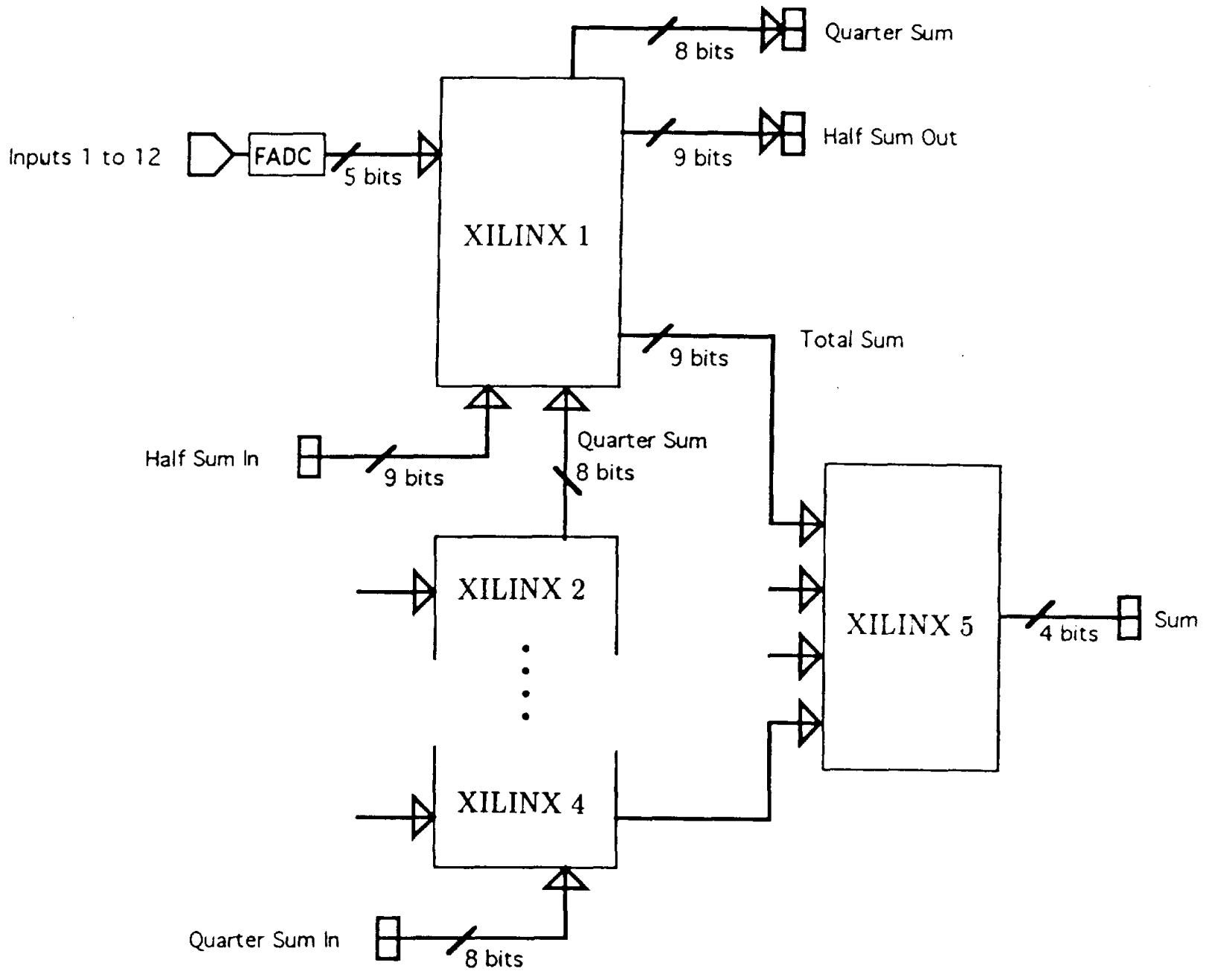

Figure 12: Trigger mixer card block diagram. Four XILINX programmable gate arrays are used as adclers to build the trigger digital partial sums. and the fifth XILINX is used as a comparator lo comstruct the trigger masks. 


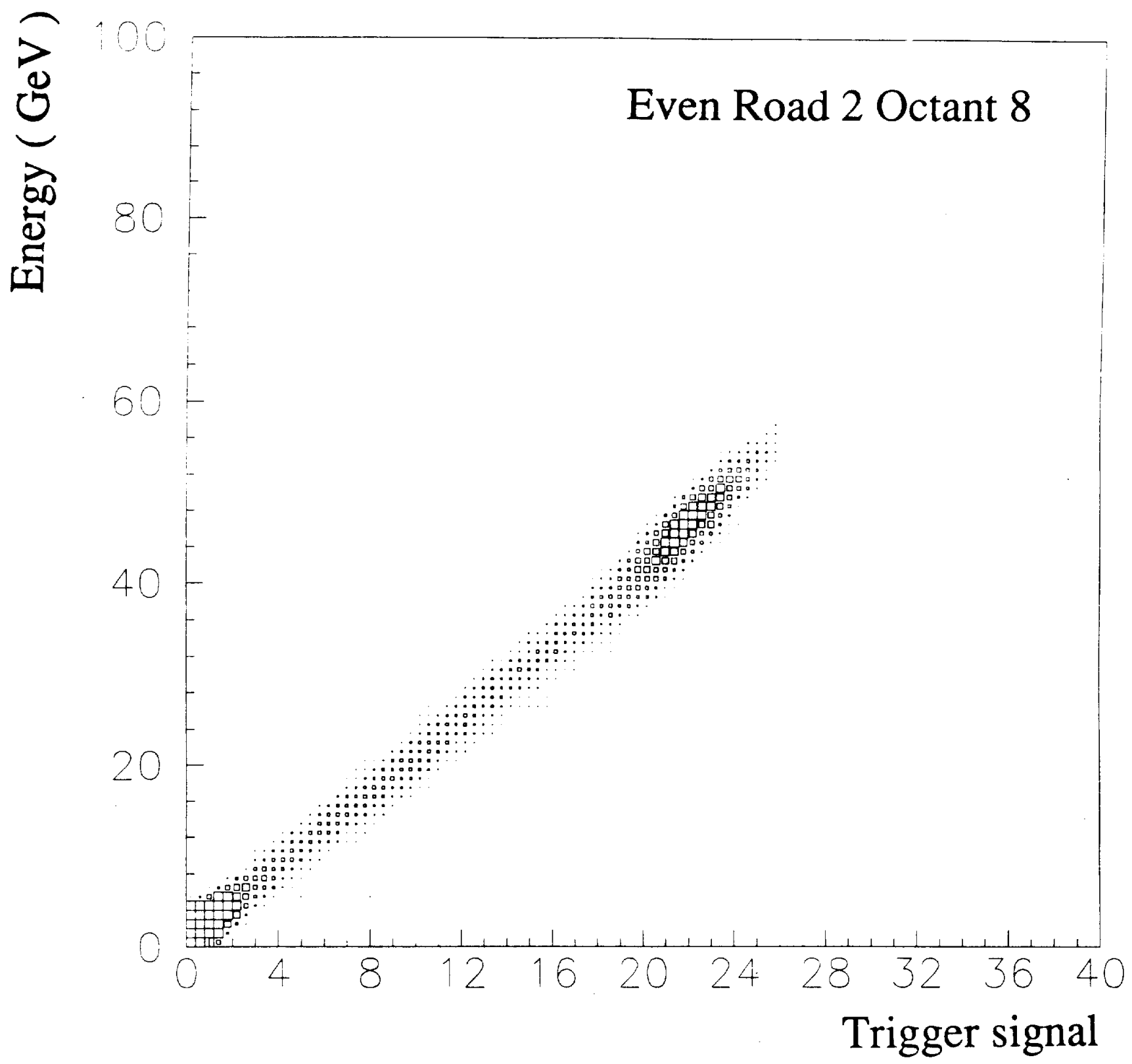

Figure 13: Trigger ignal cersus read-ont energy for a typical trigger sector. Sectors contain only onlet or even planes. so the Bhabha exents form a peak at half the beam conergy: 


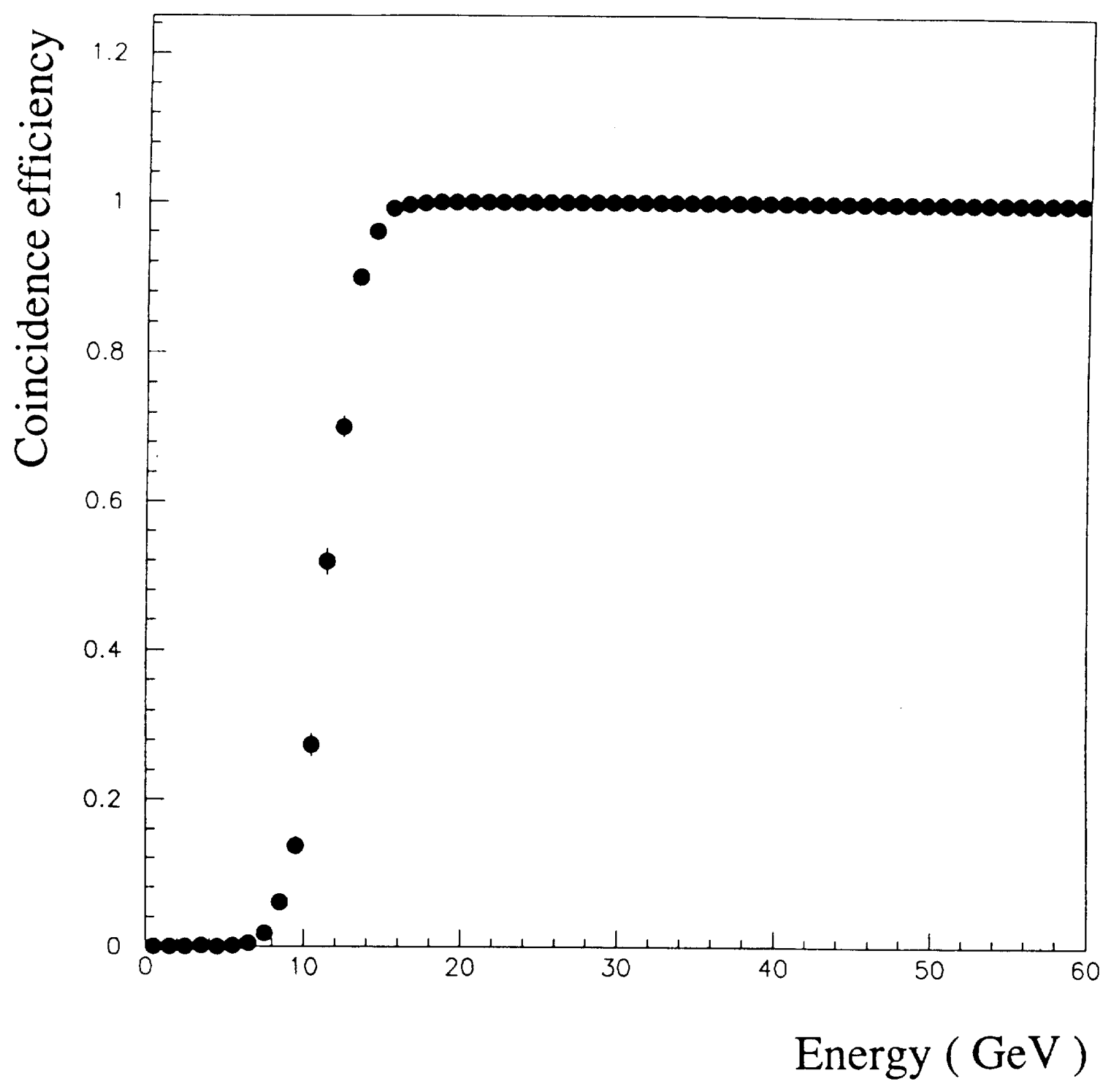

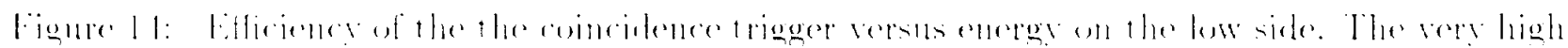

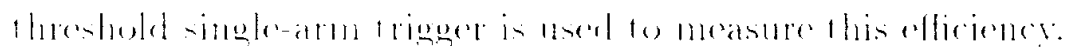



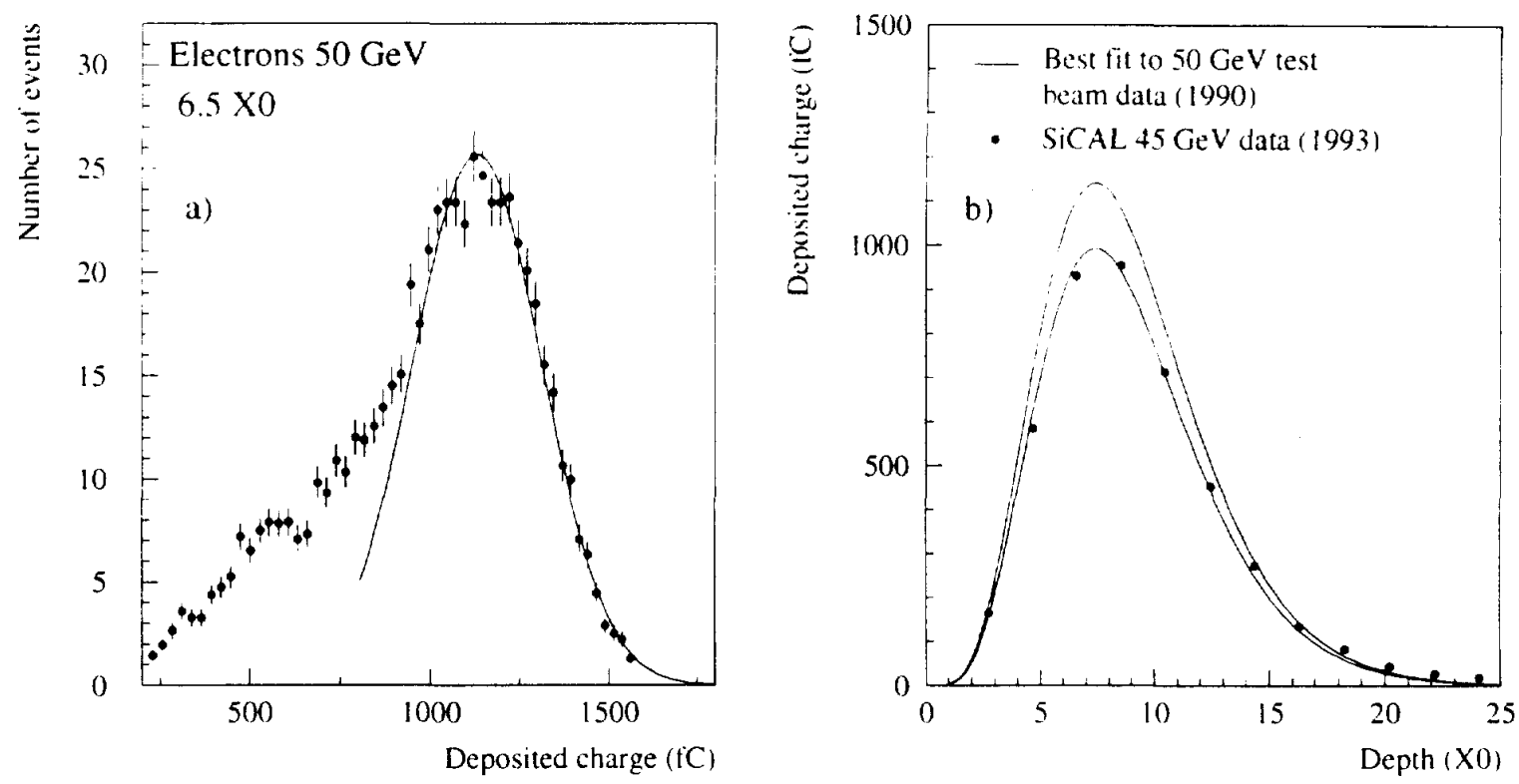

Figure 15: ('harge (fC) deposited in a single pad (shower contered) versus shower depth: (a) distribution of deposited charge at 6.5. $\mathrm{X}_{0}$, Gaussian fit gives most probable value: (b) Most probable value versus depth. smooth curve (without points) is a fit to is) (iel' - test beam prototype measurements. Points are t5 Gel Bhabha events taken at LEP. 


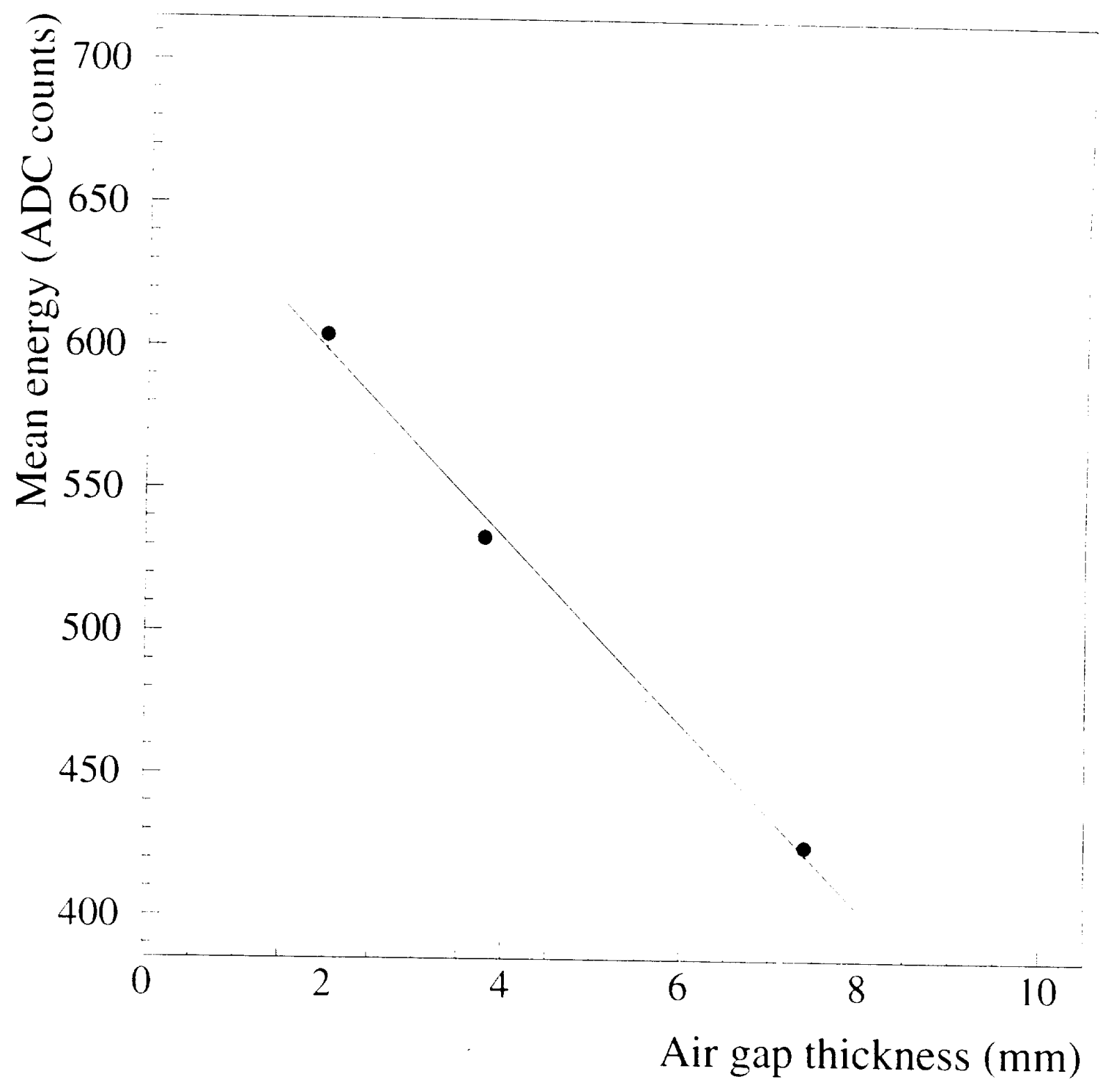

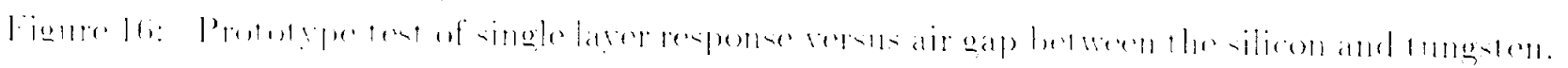




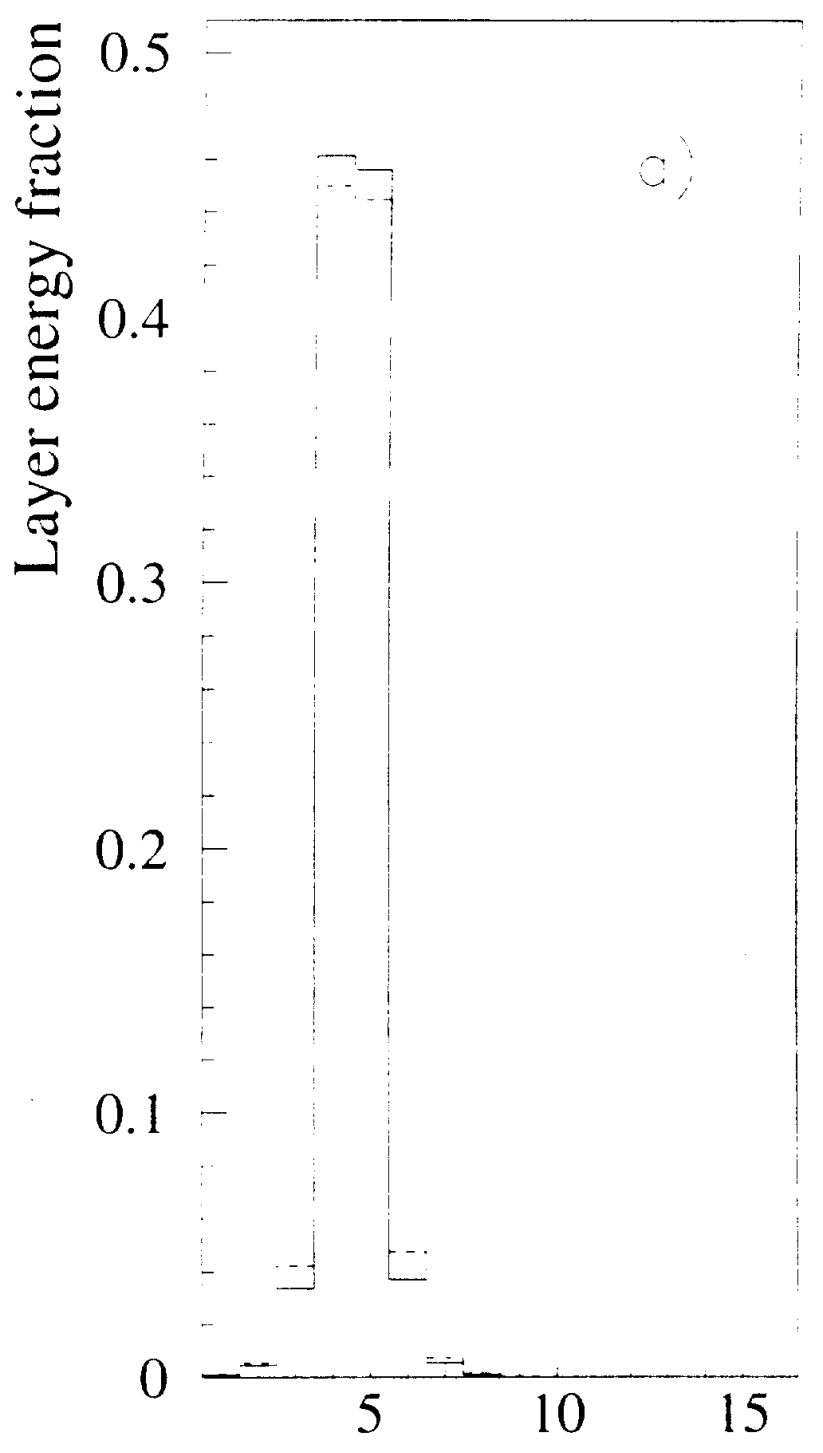

Channel number

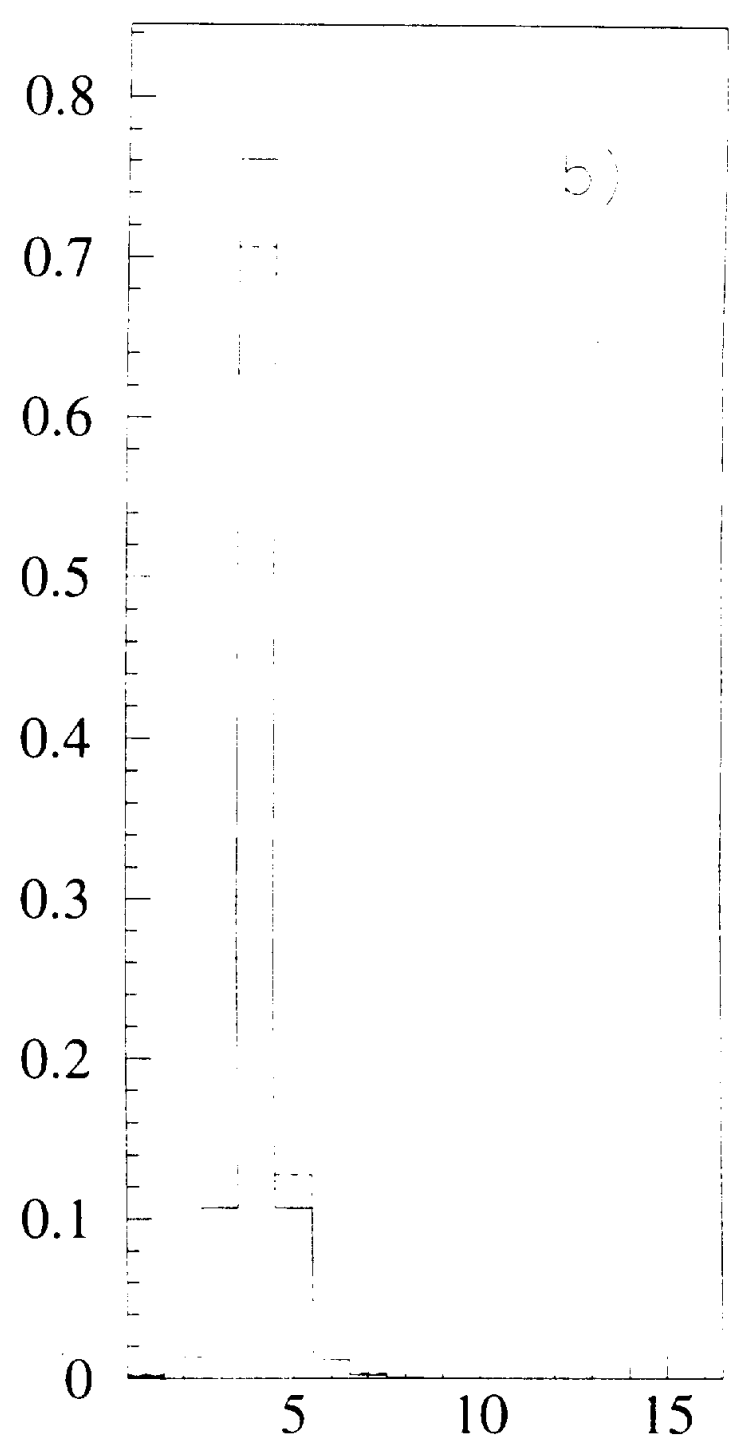

Channel number

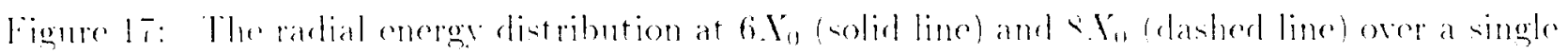

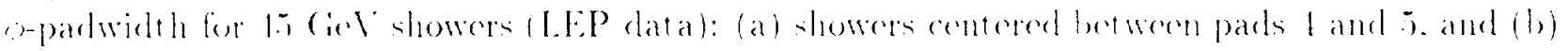
rempererl on parl 1. 


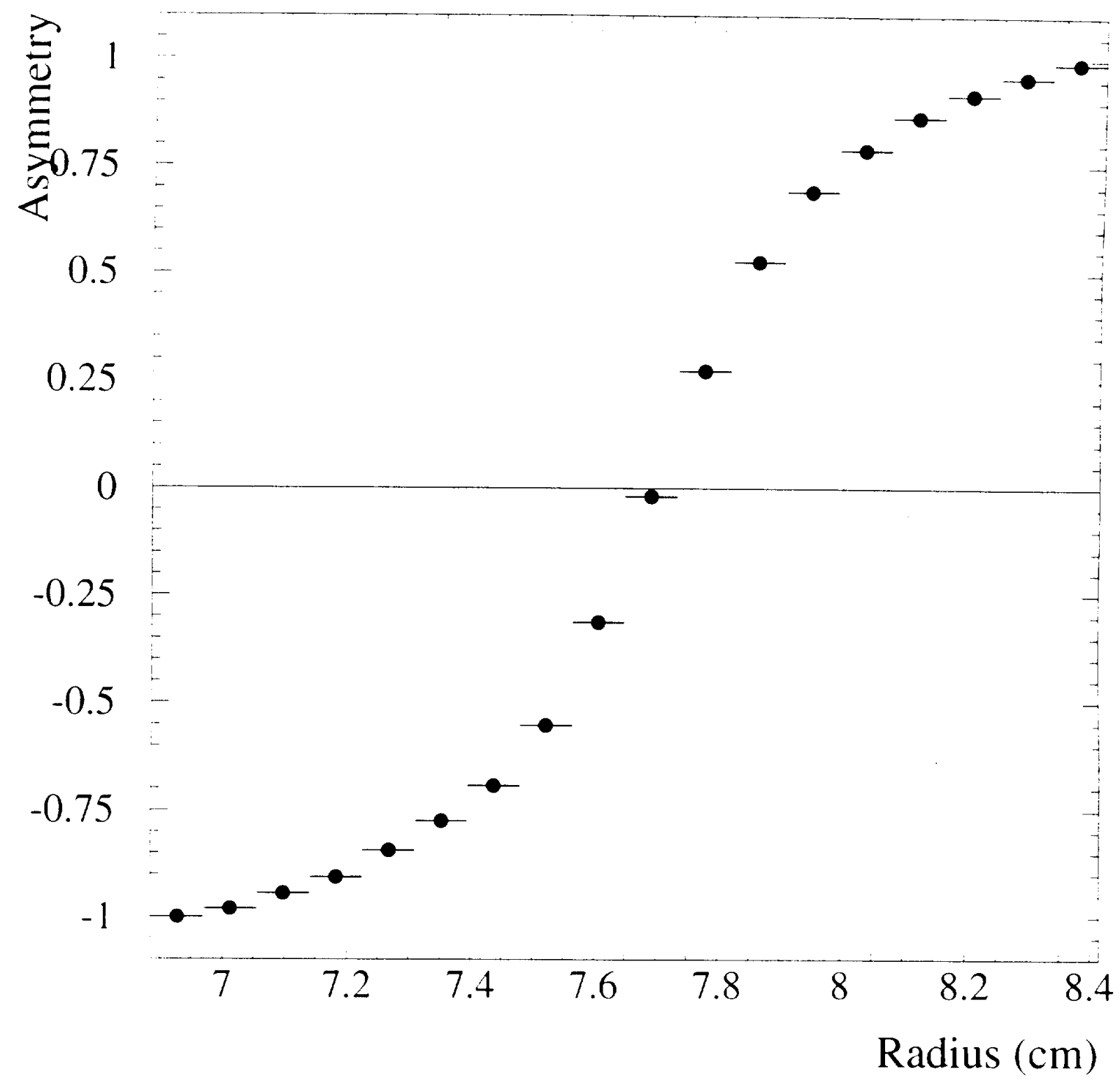

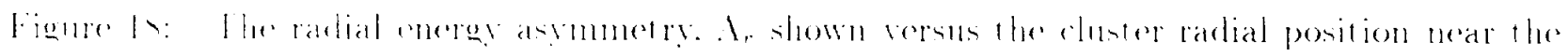
pad $3-1$ bemblame. 


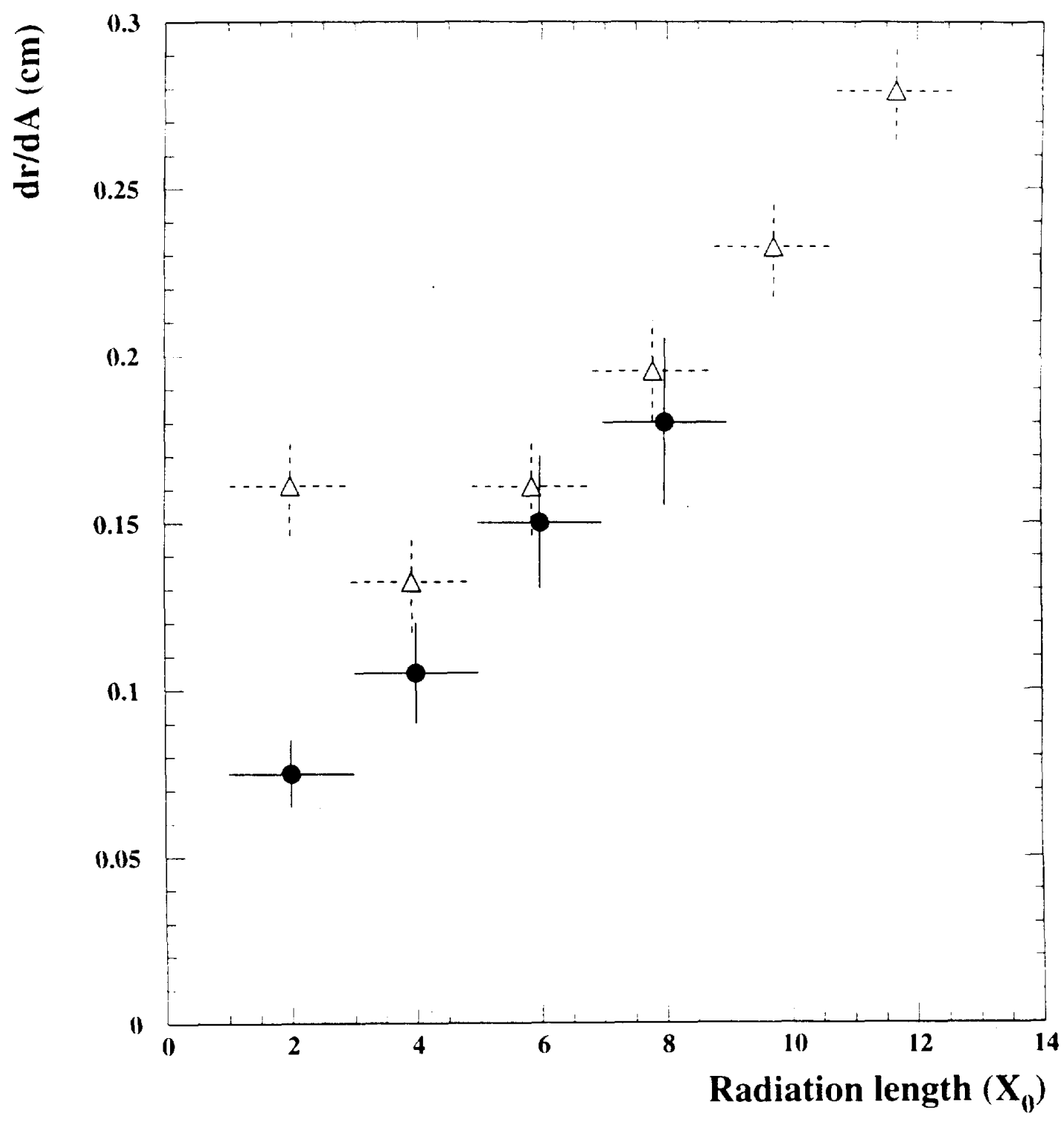

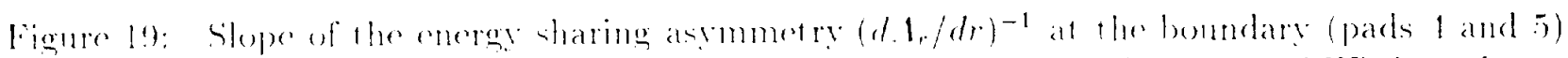

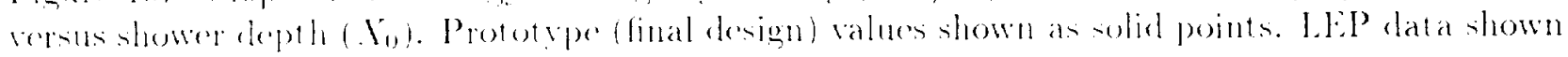
as open trialleles. 


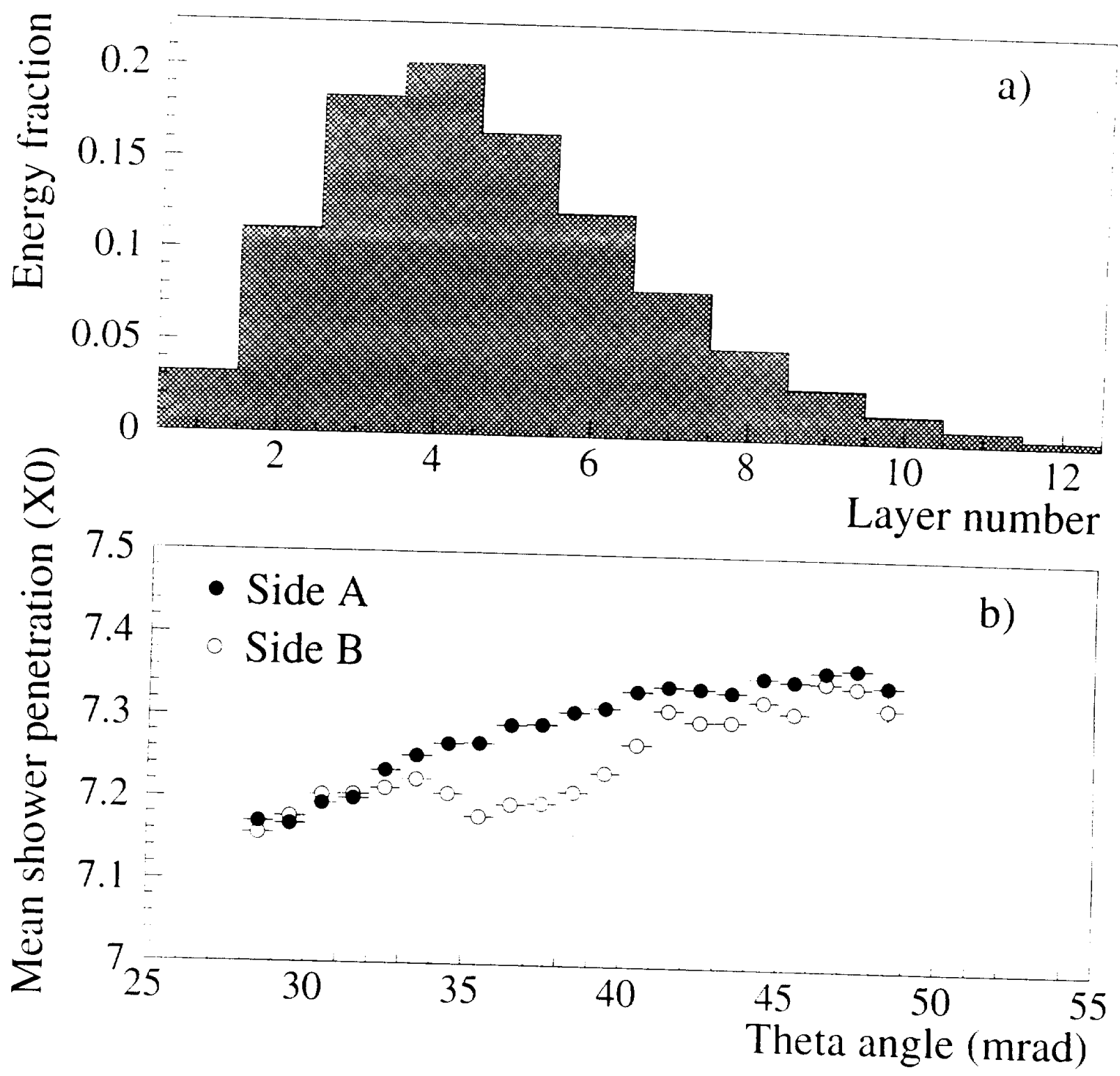

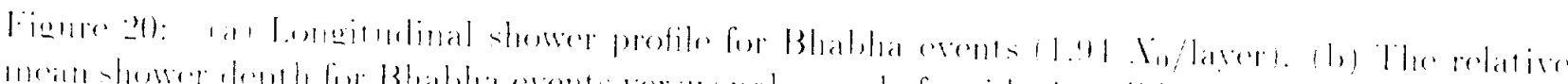



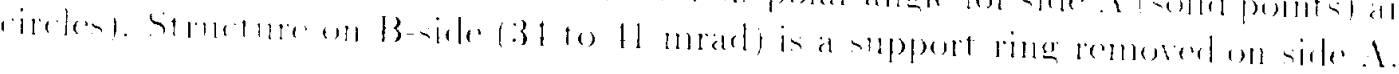




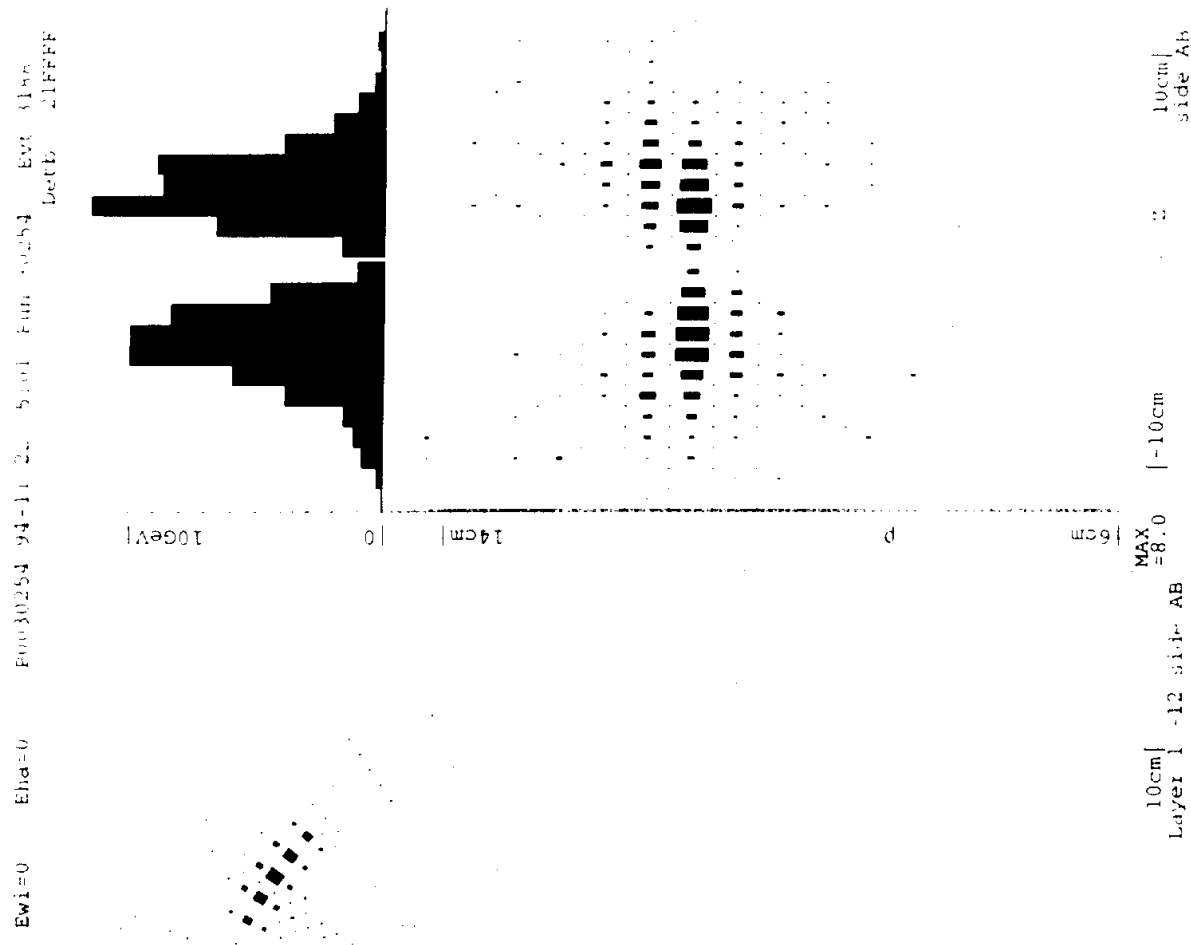

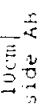

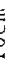

हैं

品

3

Eั

$\circ$

3

$\stackrel{E}{E}$

舀

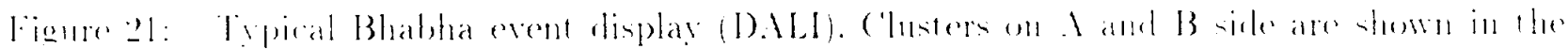

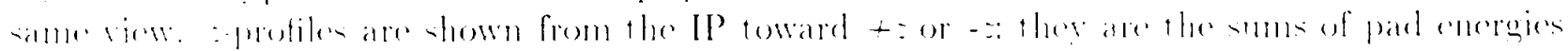
wer R-aler atch sirle. 


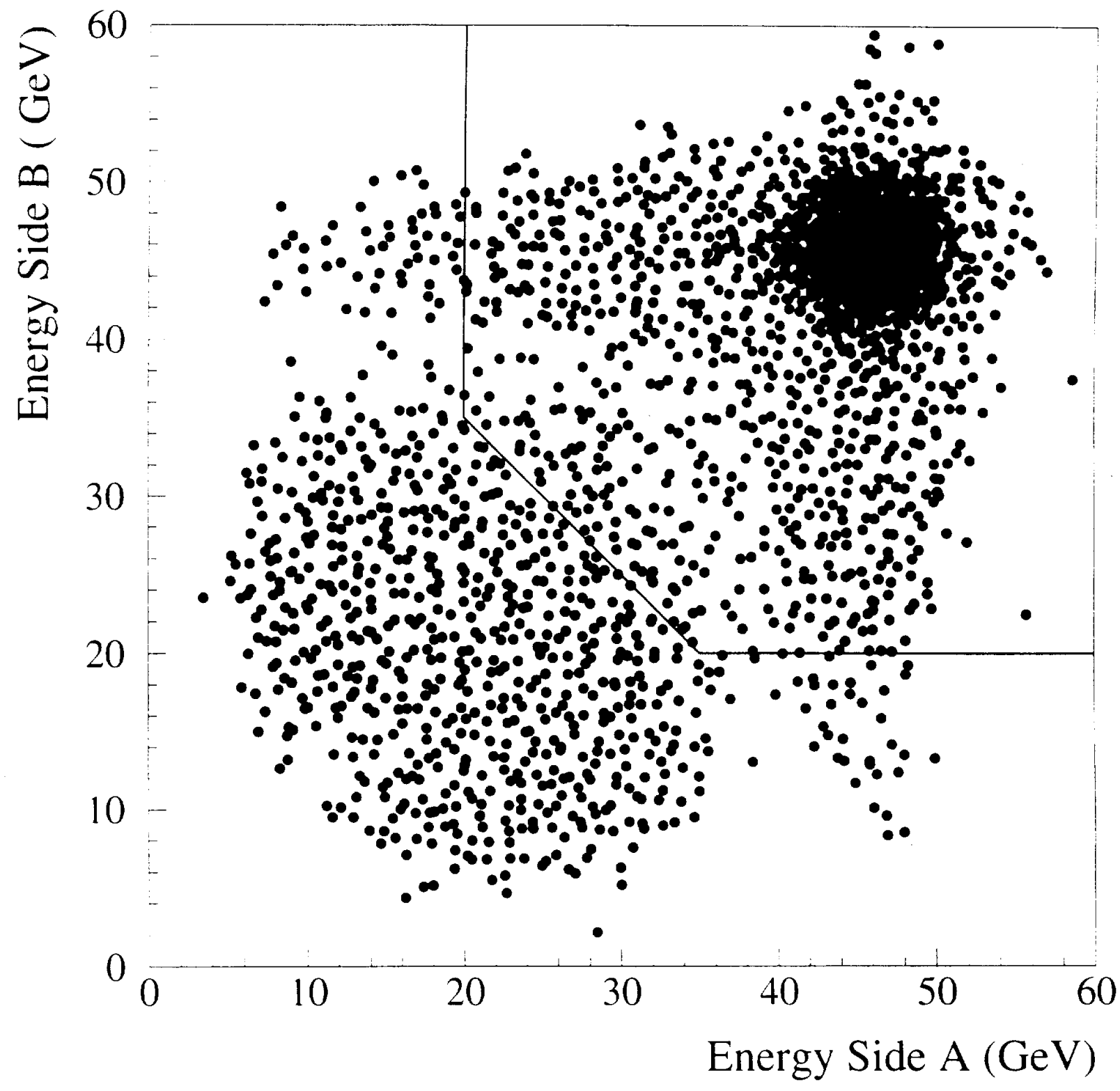

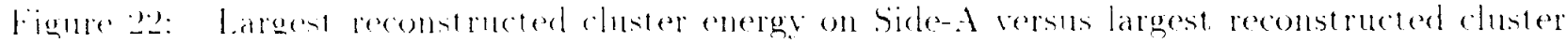
(1) 


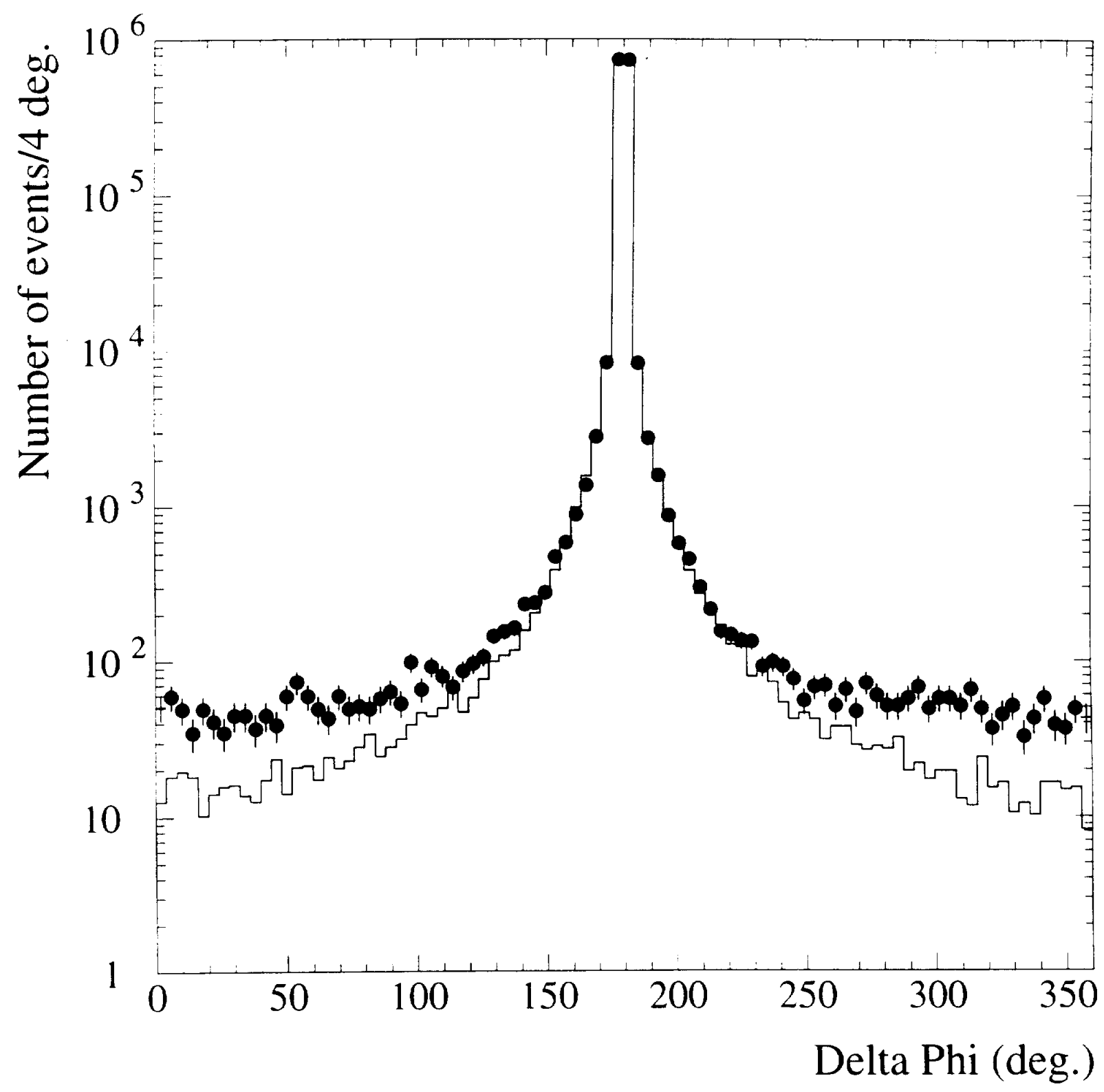

Figne 23: Distribution of the odifference Jo between the "tight-side" and "loose-side" clusters. Data are pletterlas peints and the . Ionte ('arlo (without backgromul) is shown as a line. 


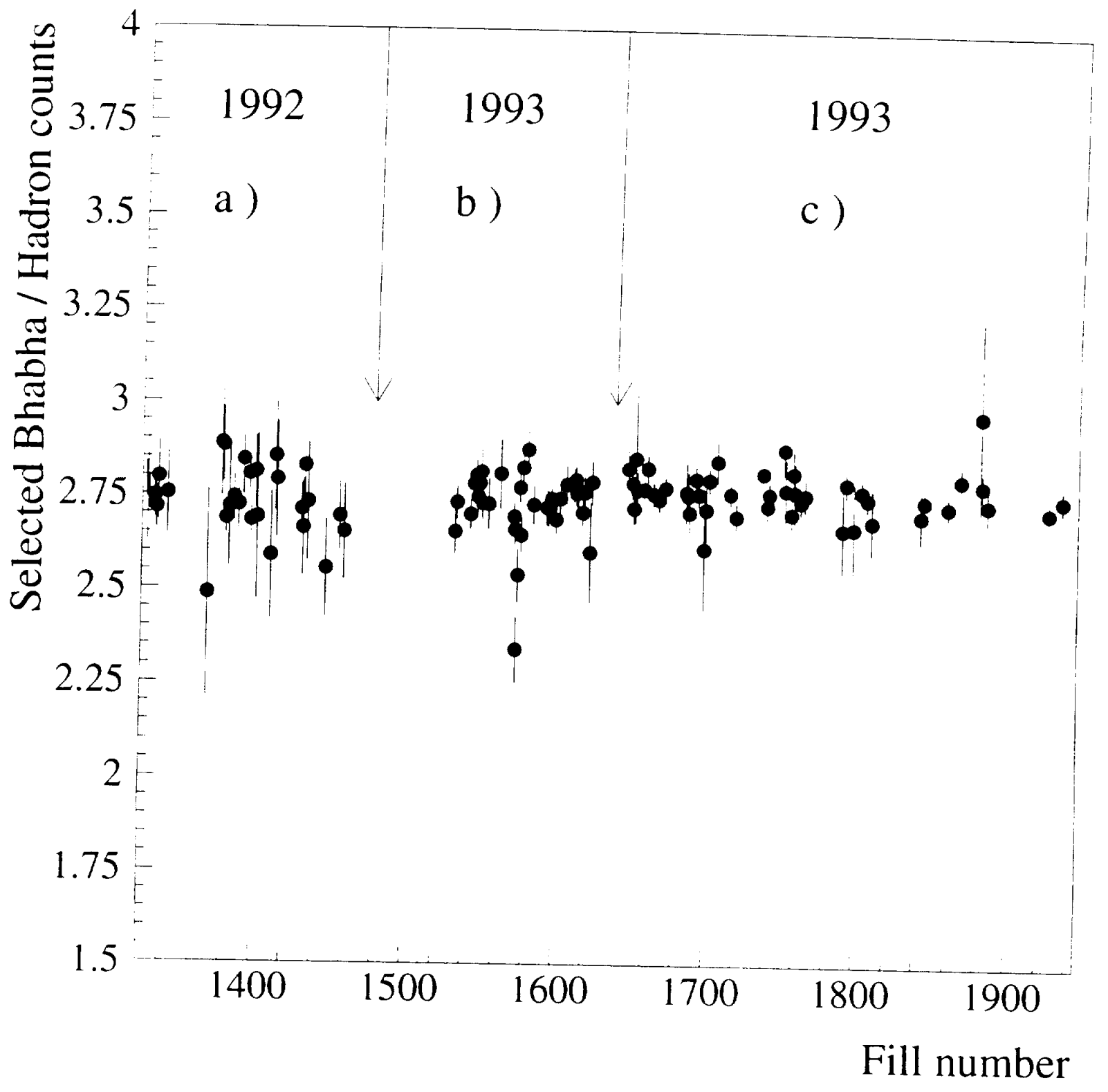

Figure 24: Ratio of background corrected Bhabhas to hadronic events taken at the Z peak (TPC and alorimeter setections averaged). ploted versus fill number: (a) two months at the end of 1992 . (b) one month prescan period of 1993 . and (c) 5 month $\%$ renonance scanning periorl of 1993.3. 
, 